University of Nebraska - Lincoln

DigitalCommons@University of Nebraska - Lincoln

Faculty Publications, Department of Mathematics

$1-1-1998$

\title{
Exponential Stability of a Thermoelastic System with Free Boundary Conditions without Mechanical Dissipation
}

\author{
George Avalos \\ University of Nebraska-Lincoln, gavalos@math.unl.edu \\ Irena Lasiecka \\ University of Virginia, il2v@Virginia.EDU
}

Follow this and additional works at: https://digitalcommons.unl.edu/mathfacpub

Part of the Mathematics Commons

Avalos, George and Lasiecka, Irena, "Exponential Stability of a Thermoelastic System with Free Boundary Conditions without Mechanical Dissipation" (1998). Faculty Publications, Department of Mathematics. 4. https://digitalcommons.unl.edu/mathfacpub/4

This Article is brought to you for free and open access by the Mathematics, Department of at DigitalCommons@University of Nebraska - Lincoln. It has been accepted for inclusion in Faculty Publications, Department of Mathematics by an authorized administrator of DigitalCommons@University of Nebraska - Lincoln. 


\title{
EXPONENTIAL STABILITY OF A THERMOELASTIC SYSTEM WITH FREE BOUNDARY CONDITIONS WITHOUT MECHANICAL DISSIPATION*
}

\author{
GEORGE AVALOS ${ }^{\dagger}$ AND IRENA LASIECKA ${ }^{\ddagger}$
}

\begin{abstract}
We show herein the uniform stability of a thermoelastic plate model with no added dissipative mechanism on the boundary (uniform stability of a thermoelastic plate with added boundary dissipation was shown in [J. LAGnese, Boundary Stabilization of Twin Plates, SIAM Stud. Appl. Math. 10, SIAM, Philadelphia, PA, 1989], as was that of the analytic case - where rotational forces are neglected - in [Z. LiU and S. ZhenG, Quarterly Appl. Math., 55 (1997), pp. 551-564]). The proof is constructive in the sense that we make use of a multiplier with respect to the coupled system involved so as to generate a fortiori the desired estimates; this multiplier is of an operator theoretic nature, as opposed to the more standard differential quantities used for related work. Moreover, the particular choice of our multiplier becomes clear only after recasting the PDE model into an associated abstract evolution equation.
\end{abstract}

Key words. thermoelastic plates, uniform stability, free boundary conditions

AMS subject classification. 35

PII. S0036141096300823

\section{Introduction.}

1.1. Statement of the problem. Let $\Omega$ be a bounded open subset of $\mathbb{R}^{2}$ with sufficiently smooth boundary $\Gamma=\Gamma_{0} \cup \Gamma_{1}, \Gamma_{0}$ and $\Gamma_{1}$ both nonempty, and $\overline{\Gamma_{0}} \cap \overline{\Gamma_{1}}=$ $\varnothing$. We consider here the following thermoelastic system taken from J. Lagnese's monograph [12]:

$$
\left\{\begin{array}{l}
\left\{\begin{array}{l}
\omega_{t t}-\gamma \Delta \omega_{t t}+\Delta^{2} \omega+\alpha \Delta \theta=0 \\
\beta \theta_{t}-\eta \Delta \theta+\sigma \theta-\alpha \Delta \omega_{t}=0
\end{array} \text { on }(0, \infty) \times \Omega ;\right. \\
\omega=\frac{\partial \omega}{\partial \nu}=0 \text { on }(0, \infty) \times \Gamma_{0} ; \\
\left\{\begin{array}{l}
\Delta \omega+(1-\mu) B_{1} \omega+\alpha \theta=0 \\
\frac{\partial \Delta \omega}{\partial \nu}+(1-\mu) \frac{\partial B_{2} \omega}{\partial \tau}-\gamma \frac{\partial \omega_{t t}}{\partial \nu}+\alpha \frac{\partial \theta}{\partial \nu}=0
\end{array} \text { on }(0, \infty) \times \Gamma_{1} ;\right. \\
\frac{\partial \theta}{\partial \nu}+\lambda \theta=0 \quad \text { on }(0, \infty) \times \Gamma, \lambda \geq 0 ; \\
\omega(t=0)=\omega^{0}, \omega_{t}(t=0)=\omega^{1}, \theta(t=0)=\theta^{0} \text { on } \Omega .
\end{array}\right.
$$

${ }^{*}$ Received by the editors March 22, 1996; accepted for publication September 30, 1996. The research of the second author was partially supported by the NSF grant DMS-9504822 and ARO grant DAAH04-96-1-0059.

http://www.siam.org/journals/sima/29-1/30082.html

$\dagger$ Institute for Mathematics and its Applications, University of Minnesota, Minneapolis, MN 554550436 (avalos@mercer.me.ttu.edu).

${ }^{\ddagger}$ Department of Applied Mathematics, Thornton Hall, University of Virginia, Charlottesville, VA 22903 (il2v@virginia.edu). 
Here, $\alpha, \beta$, and $\eta$ are strictly positive constants; positive constant $\gamma$ is proportional to the thickness of the plate and assumed to be small with $0<\gamma \leq M$; the constant $\sigma \geq 0$ and the boundary operators $B_{i}$ are given by

$$
\begin{aligned}
& B_{1} \omega \equiv 2 \nu_{1} \nu_{2} \frac{\partial^{2} \omega}{\partial x \partial y}-\nu_{1}^{2} \frac{\partial^{2} \omega}{\partial y^{2}}-\nu_{2}^{2} \frac{\partial^{2} \omega}{\partial x^{2}} ; \\
& B_{2} \omega \equiv\left(\nu_{1}^{2}-\nu_{2}^{2}\right) \frac{\partial^{2} \omega}{\partial x \partial y}+\nu_{1} \nu_{2}\left(\frac{\partial^{2} \omega}{\partial y^{2}}-\frac{\partial^{2} \omega}{\partial x^{2}}\right) ;
\end{aligned}
$$

the constant $\mu$ is the familiar Poisson's ratio $\in\left(0, \frac{1}{2}\right)$, and $\left[\nu_{1}, \nu_{2}\right]$ denotes the outward unit normal to the boundary. The given model mathematically describes a Kirchoff plate, the displacement of which is represented by the function $\omega$ subjected to a thermal damping as quantified by $\theta$. We are concerned here with the uniform stability of solutions $[\omega, \theta]$ to $(1.1)$.

1.2. Preliminaries and abstract formulation. As a departure point for obtaining the proofs of well posedness and of exponential stability, we will consider the system (1.1) as an abstract evolution equation in a certain Hilbert space, for which we introduce the following definitions and notation:

- With $H_{\Gamma_{0}}^{k}(\Omega) \equiv\left\{\omega \in H^{k}(\Omega):\left.\frac{\partial^{j} \omega}{\partial \nu^{j}}\right|_{\Gamma_{0}}=0\right.$ for $\left.j=0, \ldots, k-1\right\}$, we define $\AA: L^{2}(\Omega) \supset D(\AA) \rightarrow L^{2}(\Omega)$ to be $\AA=\Delta^{2}$, with domain

$$
\begin{aligned}
D(\AA)= & \left\{\omega \in H^{4}(\Omega) \cap H_{\Gamma_{0}}^{2}(\Omega): \Delta \omega+(1-\mu) B_{1} \omega=0 \text { on } \Gamma_{1}\right. \text { and } \\
& \left.\frac{\partial \Delta \omega}{\partial v}+(1-\mu) \frac{\partial B_{2} \omega}{\partial \tau}=0 \text { on } \Gamma_{1}\right\} .
\end{aligned}
$$

- $\AA$ is then positive definite, self-adjoint, and consequently from [8] we have the characterizations

$$
\begin{aligned}
& D\left(\AA^{\frac{1}{4}}\right)=H_{\Gamma_{0}}^{1}(\Omega) ; \\
& D\left(\AA^{\frac{1}{2}}\right)=H_{\Gamma_{0}}^{2}(\Omega) ; \\
& D\left(\AA^{\frac{3}{4}}\right)=\left\{\omega \in H^{3}(\Omega) \cap H_{\Gamma_{0}}^{2}(\Omega): \Delta \omega+(1-\mu) B_{1} \omega=0 \text { on } \Gamma_{1}\right\} .
\end{aligned}
$$

Moreover, using the Green's formula in [12], we have that for $\omega, \widehat{\omega}$ "smooth enough,"

$$
\begin{aligned}
\int_{\Omega}\left(\Delta^{2} \omega\right) \widehat{\omega} d \Omega= & a(\omega, \widehat{\omega}) \\
& +\int_{\Gamma}\left[\frac{\partial \Delta \omega}{\partial \nu}+(1-\mu) \frac{\partial B_{2} \omega}{\partial \tau}\right] \widehat{\omega} d \Gamma \\
& -\int_{\Gamma}\left[\Delta \omega+(1-\mu) B_{1} \omega\right] \frac{\partial \widehat{\omega}}{\partial \nu} d \Gamma
\end{aligned}
$$

where $a(\cdot, \cdot)$ is defined by

$$
a(\omega, \widehat{\omega}) \equiv \int_{\Omega}\left[\omega_{x x} \widehat{\omega}_{x x}+\omega_{y y} \widehat{\omega}_{y y}+\mu\left(\omega_{x x} \widehat{\omega}_{y y}+\omega_{y y} \widehat{\omega}_{x x}\right)+2(1-\mu) \omega_{x y} \widehat{\omega}_{x y}\right] d \Omega .
$$


In particular, this formula and the second characterization in (1.4) give that for all $\omega, \widehat{\omega} \in D\left(\AA^{\frac{1}{2}}\right)$,

$$
\langle\AA \hat{\omega}, \widehat{\omega}\rangle_{\left[D\left(\AA^{\frac{1}{2}}\right)\right]^{\prime} \times D\left(\AA^{\frac{1}{2}}\right)}=\left(\AA^{\frac{1}{2}} \omega, \AA^{\frac{1}{2}} \widehat{\omega}\right)_{L^{2}(\Omega)}=a(\omega, \widehat{\omega})_{L^{2}(\Omega)},
$$

and in addition,

$$
\|\omega\|_{D\left(\AA^{\frac{1}{2}}\right)}^{2}=\left\|\AA^{\frac{1}{2}} \omega\right\|_{L^{2}(\Omega)}^{2}=a(\omega, \omega) .
$$

- We define $A_{D}: L^{2}(\Omega) \supset D\left(A_{D}\right) \rightarrow L^{2}(\Omega)$ to be $A_{D}=-\Delta$, with Dirichlet boundary conditions, viz.

$$
D\left(A_{D}\right)=H^{2}(\Omega) \cap H_{0}^{1}(\Omega) .
$$

$A_{D}$ is also positive definite, self-adjoint, and, by [8],

$$
D\left(A_{D}^{\frac{1}{2}}\right)=H_{0}^{1}(\Omega) .
$$

- The space $L_{\sigma+\lambda}^{2}(\Omega)$ will be defined as

$$
L_{\sigma+\lambda}^{2}(\Omega) \equiv \begin{cases}L^{2}(\Omega) & \text { if } \sigma+\lambda>0, \\ L_{0}^{2}(\Omega) & \text { if } \sigma+\lambda=0,\end{cases}
$$

where $L_{0}^{2}(\Omega)=\left\{\theta \in L^{2}(\Omega) \ni \int_{\Omega} \theta=0\right\}$.

- We designate as $A_{R}: L^{2}(\Omega) \supset D\left(A_{R}\right) \rightarrow L^{2}(\Omega)$ the following second-order elliptic operator:

$$
\begin{aligned}
& A_{R}=-\Delta+\frac{\sigma}{\eta} \mathbf{I}, \\
& D\left(A_{R}\right)=\left\{\theta \in H^{2}(\Omega): \frac{\partial \theta}{\partial \nu}+\lambda \theta=0\right\} ;
\end{aligned}
$$

$A_{R}$ is self-adjoint, positive semidefinite on $L^{2}(\Omega)$, and, once more by [8],

$$
D\left(A_{R}^{\frac{1}{2}}\right)=H^{1}(\Omega) .
$$

When $\lambda=\sigma=0$, we shall denote the corresponding operator as $A_{N}$ (instead of as $\left.A_{R}\right)$.

Furthermore, as the bilinear form $(\nabla \theta, \nabla \widetilde{\theta})_{L^{2}(\Omega)}$ is $H^{1}(\Omega)$-elliptic on $H^{1}(\Omega) \cap$ $L_{0}^{2}(\Omega)$, we can define the norm-inducing inner product on $H^{1}(\Omega) \cap L_{\sigma+\lambda}^{2}(\Omega)$ as

$$
(\theta, \widetilde{\theta})_{H^{1}(\Omega) \cap L_{\sigma+\lambda}^{2}(\Omega)} \equiv(\nabla \theta, \nabla \widetilde{\theta})_{L^{2}(\Omega)}+\lambda(\theta, \widetilde{\theta})_{L^{2}(\Gamma)}+\frac{\sigma}{\eta}(\theta, \widetilde{\theta})_{L^{2}(\Omega)} .
$$

- $\left(\gamma_{0}, \gamma_{1}\right)$ will denote the Sobolev trace maps, which yield for $f \in C^{\infty}(\bar{\Omega})$

$$
\gamma_{0} f=\left.f\right|_{\Gamma} ; \gamma_{1} f=\left.\frac{\partial f}{\partial \nu}\right|_{\Gamma}
$$


- We define the elliptic operators $G_{1}, G_{2}$, and $D$ as thus:

$$
\begin{aligned}
& G_{1} h=v \Longleftrightarrow\left\{\begin{array}{l}
\Delta^{2} v=0 \text { in }(0, \infty) \times \Omega ; \\
v=\frac{\partial v}{\partial \nu}=0 \text { on }(0, \infty) \times \Gamma_{0} ; \\
\left\{\begin{array}{l}
\Delta v+(1-\mu) B_{1} v=h \\
\frac{\partial \Delta v}{\partial \nu}+(1-\mu) \frac{\partial B_{2} v}{\partial \tau}=0
\end{array} \text { on }(0, \infty) \times \Gamma_{1} ;\right.
\end{array}\right. \\
& G_{2} h=v \Longleftrightarrow\left\{\begin{array}{l}
\Delta^{2} v=0 \text { in }(0, \infty) \times \Omega ; \\
v=\frac{\partial v}{\partial \nu}=0 \text { on }(0, \infty) \times \Gamma_{0} ; \\
\left\{\begin{array}{l}
\Delta v+(1-\mu) B_{1} v=0 \\
\frac{\partial \Delta v}{\partial \nu}+(1-\mu) \frac{\partial B_{2} v}{\partial \tau}=h
\end{array} \text { on }(0, \infty) \times \Gamma_{1} ;\right.
\end{array}\right. \\
& D h=v \Longleftrightarrow\left\{\begin{array}{l}
\Delta v=0 \text { on }(0, \infty) \times \Omega ; \\
\left.v\right|_{\Gamma}=h \text { on }(0, \infty) \times \Gamma .
\end{array}\right.
\end{aligned}
$$

The classic regularity results of $[19$, p. 152] then provide that for $s \in \mathbb{R}$,

$$
\left\{\begin{array}{l}
D \in \mathcal{L}\left(H^{s}(\Gamma), H^{s+\frac{1}{2}}(\Omega)\right) \\
G_{1} \in \mathcal{L}\left(H^{s}\left(\Gamma_{1}\right), H^{s+\frac{5}{2}}(\Omega)\right) \\
G_{2} \in \mathcal{L}\left(H^{s}\left(\Gamma_{1}\right), H^{s+\frac{7}{2}}(\Omega)\right) .
\end{array}\right.
$$

With the operators $\AA$ and $G_{i}$ as defined above, one can readily show with the use of the Green's formula (1.5) that $\forall \omega \in D\left(\AA^{\frac{1}{2}}\right)$ the adjoints $G_{i}^{*} \AA$ $\in \mathcal{L}\left(D\left(\AA^{\frac{1}{2}}\right), L^{2}(\Gamma)\right)$ satisfy, respectively,

$$
\begin{aligned}
& G_{1}^{*} \AA \omega=\left\{\begin{array}{cc}
\left.\frac{\partial \omega}{\partial \nu}\right|_{\Gamma_{1}} & \text { on }(0, \infty) \times \Gamma_{1} ; \\
0 & \text { on }(0, \infty) \times \Gamma_{0} ;
\end{array}\right. \\
& G_{2}^{*} \AA \omega=\left\{\begin{array}{cc}
-\left.\omega\right|_{\Gamma_{1}} & \text { on }(0, \infty) \times \Gamma_{1} ; \\
0 & \text { on }(0, \infty) \times \Gamma_{0} .
\end{array}\right.
\end{aligned}
$$

- We define the operator $P_{\gamma}$ by

$$
P_{\gamma} \equiv \mathbf{I}+\gamma A_{N},
$$

and make the following points:

(i) With the parameter $\gamma>0$, we define a space $H_{\Gamma_{0}, \gamma}^{1}(\Omega)$ equivalent to $H_{\Gamma_{0}}^{1}(\Omega)$ with its inner product being

$$
\left(\omega_{1}, \omega_{2}\right)_{H_{\Gamma_{0}, \gamma}^{1}(\Omega)} \equiv\left(\omega_{1}, \omega_{2}\right)_{L^{2}(\Omega)}+\gamma\left(\nabla \omega_{1}, \nabla \omega_{2}\right)_{L^{2}(\Omega)} \quad \forall \omega_{1}, \omega_{2} \in H_{\Gamma_{0}}^{1}(\Omega),
$$


and with its dual (pivotal with respect to $L_{2}$ inner product) denoted as $H_{\Gamma_{0}, \gamma}^{-1}(\Omega)$. After recalling that $H^{1}(\Omega)=D\left(A_{N}^{1 / 2}\right)$, two extensions by continuity will then yield that

$$
\begin{aligned}
& P_{\gamma} \in \mathcal{L}\left(H_{\Gamma_{0}, \gamma}^{1}(\Omega), H_{\Gamma_{0}, \gamma}^{-1}(\Omega)\right) \text {, with } \\
& \left\langle P_{\gamma} \omega_{1}, \omega_{2}\right\rangle_{H_{\Gamma_{0}, \gamma}^{-1}(\Omega) \times H_{\Gamma_{0}, \gamma}^{1}(\Omega)}=\left(\omega_{1}, \omega_{2}\right)_{H_{\Gamma_{0}, \gamma}^{1}(\Omega)} .
\end{aligned}
$$

Furthermore, the obvious $H_{\Gamma_{0}, \gamma}^{1}(\Omega)$-ellipticity of $P_{\gamma}$ and Lax-Milgram give us that $P_{\gamma}$ is boundedly invertible, i.e.,

$$
P_{\gamma}^{-1} \in \mathcal{L}\left(H_{\Gamma_{0}, \gamma}^{-1}(\Omega), H_{\Gamma_{0}, \gamma}^{1}(\Omega)\right)
$$

and moreover, $P_{\gamma}$ being positive definite and self-adjoint as an operator $P_{\gamma}$ : $L^{2}(\Omega) \supset D\left(P_{\gamma}\right) \rightarrow L^{2}(\Omega)$, the square root $P_{\gamma}^{1 / 2}$ is consequently well defined with $D\left(P_{\gamma}^{1 / 2}\right)=H_{\Gamma_{0}, \gamma}^{1}(\Omega)$ (using the interpolation theorem in [19, p. 10]; it then follows from (1.22) and (1.24) that for $\omega$ and $\widehat{\omega} \in H_{0, \gamma}^{1}(\Omega)$,

$$
\begin{aligned}
& \left\|P_{\gamma}^{\frac{1}{2}} \omega\right\|_{L^{2}(\Omega)}^{2}=\|\omega\|_{L^{2}(\Omega)}^{2}+\gamma\|\nabla \omega\|_{L^{2}(\Omega)}^{2}=\|\omega\|_{H_{\Gamma_{0}, \gamma}^{1}(\Omega)}^{2} \\
& \left(P_{\gamma}^{\frac{1}{2}} \omega, P_{\gamma}^{\frac{1}{2} \widehat{\omega}}\right)_{L^{2}(\Omega)}=(\omega, \widehat{\omega})_{H_{\Gamma_{0}, \gamma}^{1}(\Omega)} .
\end{aligned}
$$

(ii) Finally, inasmuch as Green's formula yields for $\omega, \widehat{\omega} \in D\left(\AA^{\frac{1}{2}}\right)$,

$$
\begin{aligned}
& \gamma\left\langle\left(\Delta+\AA G_{2} \gamma_{1}\right) \omega, \widehat{\omega}\right\rangle_{H_{\Gamma_{0}, \gamma}^{-1}(\Omega) \times H_{\Gamma_{0}, \gamma}^{1}(\Omega)} \\
& =-\gamma(\nabla \omega, \nabla \widehat{\omega})_{L^{2}(\Omega)}+\gamma\left(\frac{\partial \omega}{\partial \nu}, \widehat{\omega}\right)_{L^{2}\left(\Gamma_{1}\right)}+\gamma\left(\gamma_{1} \omega, G_{2}^{*} \AA \widehat{\omega}\right)_{L^{2}\left(\Gamma_{1}\right)} \\
& =-\gamma(\nabla \omega, \nabla \widehat{\omega})_{L^{2}(\Omega)}=-\gamma\left\langle A_{N} \omega, \widehat{\omega}\right\rangle_{H_{\Gamma_{0}, \gamma}^{-1}(\Omega) \times H_{\Gamma_{0}, \gamma}^{1}(\Omega)},
\end{aligned}
$$

after using (1.20). We thus obtain after two extensions by continuity to $H_{\Gamma_{0}, \gamma}^{1}(\Omega)$ that

(1.29) $P_{\gamma}=\mathbf{I}-\gamma\left(\Delta+\AA G_{2} \gamma_{1}\right)$ as elements of $\mathcal{L}\left(H_{\Gamma_{0}, \gamma}^{1}(\Omega), H_{\Gamma_{0}, \gamma}^{-1}(\Omega)\right)$.

In obtaining the equality above, we have used implicitly the fact that for every $\varpi^{*} \in H_{\Gamma_{0}, \gamma}^{-1}(\Omega)$ and $\varpi \in D\left(\AA^{1 / 2}\right)$,

$$
\left\langle\varpi^{*}, \varpi\right\rangle_{H_{\Gamma_{0}, \gamma}^{-1}(\Omega) \times H_{\Gamma_{0}, \gamma}^{1}(\Omega)}=\left\langle\varpi^{*}, \varpi\right\rangle_{\left[D\left(\AA^{\frac{1}{2}}\right)\right]^{\prime} \times D\left(\AA^{\frac{1}{2}}\right)} .
$$

- We denote the Hilbert space $\mathbf{H}_{\gamma}$ to be

$$
\mathbf{H}_{\gamma} \equiv D\left(\AA^{\frac{1}{2}}\right) \times H_{\Gamma_{0}, \gamma}^{1}(\Omega) \times L_{\sigma+\lambda}^{2}(\Omega),
$$

with the inner product

$$
\begin{aligned}
& \left(\left[\begin{array}{l}
\omega_{1} \\
\omega_{2} \\
\theta
\end{array}\right],\left[\begin{array}{l}
\widehat{\omega}_{1} \\
\widehat{\omega}_{2} \\
\widehat{\theta}
\end{array}\right]\right)_{\mathbf{H}_{\gamma}} \\
& =\left(\AA^{\frac{1}{2}} \omega_{1}, \AA^{\frac{1}{2}} \widehat{\omega}_{1}\right)_{L^{2}(\Omega)}+\left(P_{\gamma}^{\frac{1}{2}} \omega_{2}, P_{\gamma}^{\frac{1}{2}} \widehat{\omega}_{2}\right)_{L^{2}(\Omega)}+\beta(\theta, \widehat{\theta})_{L^{2}(\Omega)} .
\end{aligned}
$$


- With the above definitions, we then set $\mathcal{A}_{\gamma}: \mathbf{H}_{\gamma} \supset D\left(\mathcal{A}_{\gamma}\right) \rightarrow \mathbf{H}_{\gamma}$ to be

$$
\begin{aligned}
& \mathcal{A}_{\gamma} \equiv\left(\begin{array}{ccc}
\mathbf{I} & \mathbf{0} & \mathbf{0} \\
\mathbf{0} & P_{\gamma}^{-1} & \mathbf{0} \\
\mathbf{0} & \mathbf{0} & \mathbf{I}
\end{array}\right)\left(\begin{array}{ccc}
0 & \mathbf{I} & 0 \\
-\AA & 0 & (\mathbf{q}) \\
0 & -\frac{\alpha}{\beta} A_{D}\left(\mathbf{I}-D \gamma_{0}\right) & -\frac{\eta}{\beta} A_{R}
\end{array}\right),
\end{aligned}
$$

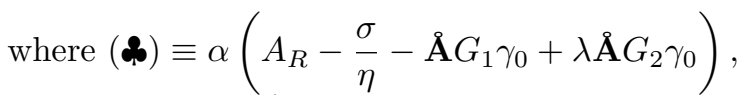

$$
\begin{aligned}
& \text { with } D\left(\mathcal{A}_{\gamma}\right)=\left\{\left[\omega_{1}, \omega_{2}, \theta\right] \in D\left(\AA^{\frac{1}{2}}\right) \times D\left(\AA^{\frac{1}{2}}\right) \times D\left(A_{R}\right) \cap L_{\sigma+\lambda}^{2}(\Omega)\right. \\
& \text { such that } \AA \omega_{1}+\alpha \AA G_{1} \gamma_{0} \theta-\alpha \lambda \AA G_{2} \gamma_{0} \theta \in H_{\Gamma_{0}, \gamma}^{-1}(\Omega) \\
& \text { and } \left.\alpha \Delta \omega_{2}+\eta \Delta \theta \in L_{\sigma+\lambda}^{2}(\Omega)\right\} \text {. }
\end{aligned}
$$

If we take the initial data $\left[\omega^{0}, \omega^{1}, \theta^{0}\right]$ to be in $\mathbf{H}_{\gamma}$, then the coupled system (1.1) becomes the operator theoretic model

$$
\begin{gathered}
\frac{d}{d t}\left[\begin{array}{c}
\omega \\
\omega_{t} \\
\theta
\end{array}\right]=\mathcal{A}_{\gamma}\left[\begin{array}{c}
\omega \\
\omega_{t} \\
\theta
\end{array}\right], \\
{\left[\begin{array}{r}
\omega(0) \\
\omega_{t}(0) \\
\theta(0)
\end{array}\right]=\left[\begin{array}{c}
\omega^{0} \\
\omega^{1} \\
\theta^{0}
\end{array}\right] .}
\end{gathered}
$$

REMARK 1. For initial data $\left[\omega^{0}, \omega^{1}, \theta^{0}\right]$ in $D\left(\mathcal{A}_{\gamma}\right)$, the two equations of $(1.1)$ may be written pointwise as

$$
\begin{aligned}
& P_{\gamma} \omega_{t t}=-\AA \omega-\alpha \AA G_{1} \gamma_{0} \theta+\alpha \lambda \AA G_{2} \gamma_{0} \theta-\alpha \Delta \theta \text { in } H_{\Gamma_{0}, \gamma}^{-1}(\Omega) ; \\
& \beta \theta_{t}=\eta \Delta \theta-\sigma \theta+\alpha \Delta \omega_{t} \text { in } L_{\sigma+\lambda}^{2}(\Omega) .
\end{aligned}
$$

1.3. Previous literature. In recent years, questions related to the controllability and stabilization of thermoelastic plates have drawn considerable attention in the recent past (see [10], [12], [9], [21], [22], and [24]); we shall concentrate here on detailing results of strong and uniform stability related to the present model, that of the two-dimensional Kirchoff plate coupled with the heat equation. This particular model, associated with free boundary conditions, was introduced by J. Lagnese in [12]. In that work, he established the well posedness and exponential stability of (1.1) with $\gamma$ strictly positive, and with the appropriately chosen feedback mechanisms $\left[\mathcal{F}_{1}\left(\omega_{t}\right), \mathcal{F}_{2}\left(\omega_{t}\right)\right]$ inserted into the natural boundary conditions of the Kirchoff plate component of the system, viz.

$$
\left\{\begin{array}{l}
\omega=\frac{\partial \omega}{\partial \nu}=0 \text { on }(0, \infty) \times \Gamma_{0} \\
\Delta \omega+(1-\mu) B_{1} \omega+\alpha \theta=\mathcal{F}_{1}\left(\omega_{t}\right) \text { on }(0, \infty) \times \Gamma_{1}, \\
\frac{\partial \Delta \omega}{\partial \nu}+(1-\mu) \frac{\partial B_{2} \omega}{\partial \tau}-\gamma \frac{\partial \omega_{t t}}{\partial \nu}+\alpha \frac{\partial \theta}{\partial \nu}=\mathcal{F}_{2}\left(\omega_{t}\right) \text { on }(0, \infty) \times \Gamma_{1}
\end{array}\right.
$$


the proof of Lagnese is based on the use of differential multipliers, and it exploits the fact that $\gamma>0$. Since, from a physical point of view, the thermal effects present should induce some measure of energy dissipation (in fact, one can show the homogeneous system's strong stability by routine methods; see [12, Chap. 7], including the remark at the end of sect. 2.3 on p. 161), a natural question arising in this context is whether the system is actually (uniformly) stable without the boundary feedbacks $\mathcal{F}_{1}\left(\omega_{t}\right)$, $\mathcal{F}_{2}\left(\omega_{t}\right)$ in place, i.e., when there are no added mechanical forces. Indeed, in the case $\gamma=0$ and with different boundary conditions than those in (1.1) imposed upon the system, the answer to the question is in the affirmative and has been provided by several authors. With $\gamma=0$, J. Kim in [10] showed the uniform stability of (1.1) with the clamped boundary conditions $\omega=\frac{\partial \omega}{\partial \nu}=\theta=0$ on $\Gamma$, as did J. Rivera and R. Racke in [25], who studied the coupled equation with the hinged boundary conditions $\omega=\Delta \omega=\theta=0$. Also with $\gamma=0$, Z. Liu and S. Zheng in [21] proved the exponential stability of (1.1) with the boundary conditions

$$
\left\{\begin{array}{l}
\omega=\frac{\partial \omega}{\partial \nu}=0 \text { on }(0, \infty) \times \Gamma_{0}, \\
\omega=\Delta \omega+(1-\mu) B_{1} \omega+\alpha \theta=0 \text { on }(0, \infty) \times \Gamma_{1},
\end{array}\right.
$$

leaving the case of free boundary conditions as an open question, even in the case $\gamma=0$. The proof of Liu and Zheng is indirect in the sense that it is based on a contradiction argument applied to the exponential decay stability criterion (due to L. Monauni, R. Nagel, and F. Huang), a criterion essentially dictating the uniform estimate for that part of the resolvent which lies on the imaginary axis. On the other hand, it is now known that the case $\gamma=0$ is rather special as the corresponding system (at least for certain boundary conditions) generates an analytic semigroup (see [20]), a consequence of which will be the exponential stability of the system (recall that the system is strongly stable). Given these results, the question of interest now is whether the given thermoelastic system (without any additional boundary dissipation) is uniformly stable in the nonanalytic case, viz. $\gamma>0$, with consequently the elastic part of the system being of hyperbolic character.

A partial answer to the question above was given by the present authors in [2], [3]: with $\gamma>0$ in (1.1) and the boundary conditions

$$
\left\{\begin{array}{l}
\omega=(1-\chi) \frac{\partial \omega}{\partial \nu}=0 \\
\chi\left(\Delta \omega+(1-\mu) B_{1} \omega\right)+\alpha \theta=0
\end{array} \quad \text { on }(0, \infty) \times \Gamma\right.
$$

replacing the higher order ones for $\omega$ which are being considered in this work, where the parameter $\chi$ above is either 0 or 1 , it is shown that the partial differential equation is uniformly stable with decay estimates which are "robust" with respect to the parameter $\gamma$. The proof of this stability result is through an implementation of the multiplier method (see [11] a for treatise of this technique), with an operator theoretic quantity taken as the particular multiplier of choice.

The main goal of the present paper is to provide an affirmative answer to the question of uniform stability of (1.1) with the free boundary conditions in place, again with $\gamma>0$. The fact that the presence of these higher order boundary conditions greatly complicates the analysis was duly noted in [21], and the arguments employed in that work do not carry over for plates with free boundary conditions, even when $\gamma=0$. 
Like our earlier work in [2], [3], the proof of uniform decay here is "direct," based on pseudodifferential (or operator theoretic) multipliers, in contrast to the contradiction argument supplied in [21] for the case $\gamma=0$ and clamped boundary conditions. In addition, our direct proof, making use as it does of the multiplier method, carries the advantage of providing explicit estimates of the decay rates. However, an application of the multiplier method alone is not enough to obtain the desired inequalities for the equation (1.1) in the case when free boundary conditions are present. Indeed, in proving the stability result (Theorem 1.3 below) we must couple the use of an operator theoretic multiplier with a decomposition of the solution $\omega$ into three separate components, and a subsequent and crucial invocation of recently derived trace regularity results to handle each of these in distinct fashion; in particular, we exploit the observation that the time derivative of one of these components (modulo a change of variable) solves a certain wave equation. This scrutiny of boundary traces for the hyperbolic component $\omega$ of the dynamics is a sine qua non for obtaining the necessary estimates for uniform decay. Finally, we must emphasize that the acute difficulty of the problem which necessitates the use of microlocally derived trace estimates is owing solely to the specific boundary conditions being considered here and does not appear for other combinations of lower order boundary conditions.

1.4. Statement of the results. We shall begin by giving preliminary results regarding the well posedness of the system (1.1) and the regularity of its solutions.

THEOREM 1.1 (well posedness). Again with the parameter $\gamma>0, \mathcal{A}_{\gamma}$, given by (1.33), generates a $C_{0}$-semigroup of contractions $\left\{e^{\mathcal{A}_{\gamma} t}\right\}_{t \geq 0}$ on the energy space $\boldsymbol{H}_{\gamma}$; therefore for initial data $\left[\omega^{0}, \omega^{1}, \theta^{0}\right]$ in $\boldsymbol{H}_{\gamma}$, the solution $\left[\omega, \omega_{t}, \theta\right]$ to $(1.34)$, and consequently to (1.1), is given by

$$
\left[\begin{array}{c}
\omega \\
\omega_{t} \\
\theta
\end{array}\right]=e^{\mathcal{A}_{\gamma}(\cdot)}\left[\begin{array}{c}
\omega^{0} \\
\omega^{1} \\
\theta^{0}
\end{array}\right] \in C\left([0, T], \boldsymbol{H}_{\gamma}\right) .
$$

The following regularity result is needed to justify the computations performed below.

THEOREM 1.2. For initial data $\left[\omega^{0}, \omega^{1}, \theta^{0}\right] \in D\left(\mathcal{A}_{\gamma}^{2}\right)$, we have the following:

(i) the solution $\left[\omega, \omega_{t}, \theta\right]$ to $(1.1)$ is an element of $C\left([0, T] ; H^{4}(\Omega) \times H^{3}(\Omega) \times\right.$ $\left.H^{2}(\Omega)\right)$.

(ii) $\omega-\gamma G_{2} \gamma_{1} \omega_{t t}+\alpha G_{1} \gamma_{0} \theta-\alpha \lambda G_{2} \gamma_{0} \theta \in C([0, T] ; D(\AA))$.

Our main result is as follows.

THEOREM 1.3 (uniform stability). With $\gamma>0$, the solution $\left[\omega, \omega_{t}, \theta\right]$ of $(1.1)$ decays exponentially; that is to say, there exist constants $\delta>0$ and $M_{\delta} \geq 1$ such that for all $t>0$,

$$
\left\|\left[\begin{array}{c}
\omega(t) \\
\omega_{t}(t) \\
\theta(t)
\end{array}\right]\right\|_{\boldsymbol{H}_{\gamma}} \leq M_{\delta} e^{-\delta t}\left\|\left[\begin{array}{c}
\omega^{0} \\
\omega^{1} \\
\theta^{1}
\end{array}\right]\right\|_{\boldsymbol{H}_{\gamma}} .
$$

REMARK 2. The estimates obtained in Theorem 1.3 are not uniform with respect to the parameter $\gamma>0$. Indeed, the arguments used in the proof break down when $\gamma=0$, and consequently the estimates leading to the statement in Theorem 1.3 blow up when $\gamma \rightarrow 0$. This is due to technicalities of the proof which rely on the strict hyperbolicity of the model (a property which is lost in the limit case $\gamma=0$ ). On the other hand, in the case $\gamma=0$, it has been recently shown in [27] that the thermoelastic 
system with free boundary conditions generates an analytic semigroup. Therefore, a posteriori (recalling the strong stability of the system), we conclude that uniform stability holds true also for the case $\gamma=0$. However, these estimates cannot be reconstructed as a limiting case of the present problem when $\gamma>0$. This is unlike the case of other boundary conditions associated with this model (see [3]).

2. Proofs. The proofs of well posedness and of regularity (Theorems 1.1 and 1.2) are by now fairly routine (see [12, Chap. 7] for related well posedness/regularity results). However, since these preliminaries are critical for our ultimate end of uniform stability, we provide their concise proofs for the sake of completeness.

2.1. Proof of Theorem 1.1. In establishing the semigroup generation of $\mathcal{A}_{\gamma}$, we will show that the conditions of the Lumer-Phillips theorem are satisfied; namely, we demonstrate here that $\mathcal{A}_{\gamma}$ is maximal dissipative.

To show the dissipativity of $\mathcal{A}_{\gamma}$ : for $\left[\omega_{1}, \omega_{2}, \theta\right] \in D\left(\mathcal{A}_{\gamma}\right)$ we have

$$
\begin{aligned}
& \left(\mathcal{A}_{\gamma}\left[\begin{array}{c}
\omega_{1} \\
\omega_{2} \\
\theta
\end{array}\right],\left[\begin{array}{c}
\omega_{1} \\
\omega_{2} \\
\theta
\end{array}\right]\right)_{\mathbf{H}_{\gamma}} \\
& =\left(\AA^{\frac{1}{2}} \omega_{2}, \AA^{\frac{1}{2}} \omega_{1}\right)_{L^{2}(\Omega)} \\
& +\left(P_{\gamma}^{\frac{1}{2}} P_{\gamma}^{-1}\left(-\AA \omega_{1}+\alpha A_{R} \theta-\frac{\alpha \sigma}{\eta} \theta-\alpha \AA G_{1} \gamma_{0} \theta+\alpha \lambda \AA G_{2} \gamma_{0} \theta\right), P_{\gamma}^{\frac{1}{2}} \omega_{2}\right)_{L^{2}(\Omega)} \\
& -\alpha\left(A_{D}\left(\mathbf{I}-D \gamma_{0}\right) \omega_{2}, \theta\right)_{L^{2}(\Omega)}-\left(\eta A_{R} \theta, \theta\right)_{L^{2}(\Omega)} ;
\end{aligned}
$$

using the characterizations in (1.4) and (1.20), along with the equality posted in (2.23), we have upon the taking of adjoints that

$$
\begin{aligned}
(2.1)= & \left(\AA^{\frac{1}{2}} \omega_{2}, \AA^{\frac{1}{2}} \omega_{1}\right)_{L^{2}(\Omega)}-\left\langle\AA \omega_{1}, \omega_{2}\right\rangle_{\left[D\left(\AA^{\frac{1}{2}}\right)\right]^{\prime} \times D\left(\AA^{\frac{1}{2}}\right)} \\
& +\alpha\left(A_{R} \theta-\frac{\sigma}{\eta} \theta, \omega_{2}\right)_{L^{2}(\Omega)}-\alpha\left(\theta, \frac{\partial \omega_{2}}{\partial \nu}\right)_{L^{2}\left(\Gamma_{1}\right)}-\alpha \lambda\left(\theta, \omega_{2}\right)_{L^{2}\left(\Gamma_{1}\right)} \\
& -\alpha\left(A_{D}\left(\mathbf{I}-D \gamma_{0}\right) \omega_{2}, \theta\right)_{L^{2}(\Omega)}-\left(\eta A_{R} \theta, \theta\right)_{L^{2}(\Omega)} \\
= & \left(\AA^{\frac{1}{2}} \omega_{2}, \AA^{\frac{1}{2}} \omega_{1}\right)_{L^{2}(\Omega)}-\left(\AA^{\frac{1}{2}} \omega_{1}, \AA^{\frac{1}{2}} \omega_{2}\right)_{L^{2}(\Omega)}-\alpha\left(\Delta \theta, \omega_{2}\right)_{L^{2}(\Omega)} \\
& -\alpha\left(\theta, \frac{\partial \omega_{2}}{\partial \nu}\right)_{L^{2}\left(\Gamma_{1}\right)}-\alpha \lambda\left(\theta, \omega_{2}\right)_{L^{2}\left(\Gamma_{1}\right)}+\alpha\left(\Delta \omega_{2}, \theta\right)_{L^{2}(\Omega)}+(\eta \Delta \theta-\sigma \theta, \theta)_{L^{2}(\Omega)} \\
= & \left(\AA^{\frac{1}{2}} \omega_{2}, \AA^{\frac{1}{2}} \omega_{1}\right)_{L^{2}(\Omega)}-\left(\AA^{\frac{1}{2}} \omega_{1}, \AA^{\frac{1}{2}} \omega_{2}\right)_{L^{2}(\Omega)}+\alpha\left(\nabla \theta, \nabla \omega_{2}\right)_{L^{2}(\Omega)} \\
& -\alpha\left(\nabla \omega_{2}, \nabla \theta\right)_{L^{2}(\Omega)}-\eta\|\nabla \theta\|_{L^{2}(\Omega)}^{2}-\lambda \eta\|\theta\|_{L^{2}(\Gamma)}^{2}-\sigma\|\theta\|_{L^{2}(\Omega)}^{2}
\end{aligned}
$$

$(2.2) \leq 0$

(here, we are using the fact that $\frac{\partial \theta}{\partial \nu}=-\lambda \theta$ ); i.e., $\mathcal{A}_{\gamma}$ is dissipative. 
To show the maximality of $\mathcal{A}_{\gamma}$ : if for some $\xi>0$ and arbitrary $\left[f_{1}, f_{2}, f_{3}\right] \in \mathbf{H}_{\gamma}$, $\left[\omega_{1}, \omega_{2}, \theta\right] \in D\left(\mathcal{A}_{\gamma}\right)$ solves the equation

$$
\left(\xi \mathbf{I}-\mathcal{A}_{\gamma}\right)\left[\begin{array}{l}
\omega_{1} \\
\omega_{2} \\
\theta
\end{array}\right]=\left[\begin{array}{l}
f_{1} \\
f_{2} \\
f_{3}
\end{array}\right]
$$

then this relation holds if and only if

$$
\begin{aligned}
& \left\{\begin{array}{l}
\xi \omega_{1}-\omega_{2}=f_{1} \text { in } D\left(\AA^{\frac{1}{2}}\right), \\
\xi \omega_{2}+P_{\gamma}^{-1}\left(\AA \omega_{1}+\alpha \AA G_{1} \gamma_{0} \theta-\alpha \lambda \AA G_{2} \gamma_{0} \theta-\alpha A_{R} \theta+\frac{\alpha \sigma}{\eta} \theta\right)=f_{2} \text { in } H_{\Gamma_{0}, \gamma}^{1}(\Omega), \\
\xi \theta+\frac{\alpha}{\beta} A_{D}\left(\mathbf{I}-D \gamma_{0}\right) \omega_{2}+\frac{\eta}{\beta} A_{R} \theta=f_{3} \text { in } L_{\sigma+\lambda}^{2}(\Omega)
\end{array}\right.
\end{aligned}
$$

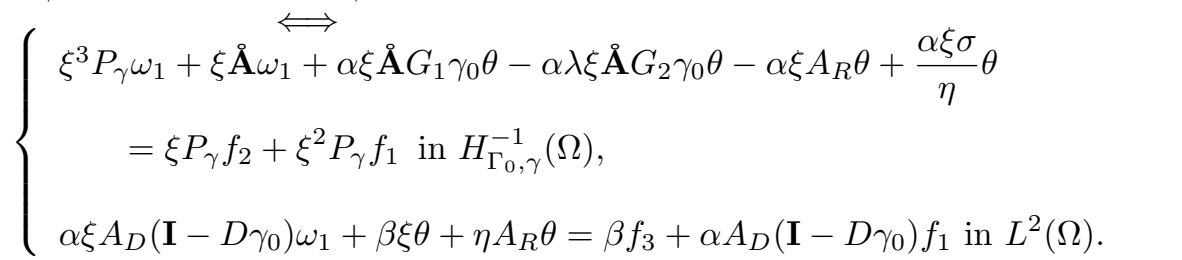

At this point we bring forth the following proposition.

Proposition 2.1. The operator $\boldsymbol{F}$ defined by

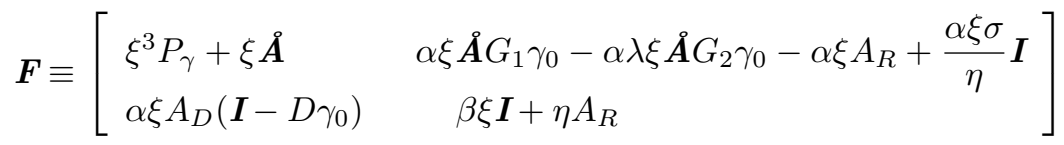

is an element of $\mathcal{L}\left(D\left(\AA^{1 / 2}\right) \times H^{1}(\Omega) \cap L_{\sigma+\lambda}^{2}(\Omega),\left[D\left(\AA^{1 / 2}\right)\right]^{\prime} \times\left[H^{1}(\Omega) \cap L_{\sigma+\lambda}^{2}(\Omega)\right]^{\prime}\right)$ and is boundedly invertible.

Proof of Proposition 2.1. Easily, from the definitions of the operators which make up the components of $\mathbf{F}$, all of which are given in section 1.2, we deduce that $\mathbf{F}$ is bounded in the asserted topology. Moreover, we note by Green's theorem that for arbitrary $\theta \in D\left(A_{R}\right)$ and $\omega \in D\left(\AA^{1 / 2}\right)$,

$$
\left\langle A_{R} \theta+\alpha \lambda \xi \AA G_{2} \gamma_{0}, \omega\right\rangle_{\left[D\left(\AA^{\frac{1}{2}}\right)\right]^{\prime} \times D\left(\AA^{\frac{1}{2}}\right)}=(\nabla \theta, \nabla \omega)_{L^{2}(\Omega)}+\frac{\sigma}{\eta}(\theta, \omega)_{L^{2}(\Omega)} ;
$$

the characterization (1.13) and an extension by continuity will then have that (2.7) holds for all $\theta$ in $H^{1}(\Omega)$, and so for $\theta$ in $H^{1}(\Omega) \cap L_{\sigma+\lambda}^{2}(\Omega)$. (2.7) in turn, when coupled with (2.23), (1.24), (1.14), (1.20), and Green's formula will provide the following coercivity inequality for all $[\omega, \theta] \in D\left(\AA^{1 / 2}\right) \times H^{1}(\Omega) \cap L_{\sigma+\lambda}^{2}(\Omega)$ :

$$
\begin{aligned}
\left\langle\mathbf{F}\left[\begin{array}{c}
\omega \\
\theta
\end{array}\right],\left[\begin{array}{c}
\omega \\
\theta
\end{array}\right]\right\rangle= & \xi^{3}\|\omega\|_{L^{2}(\Omega)}^{2}+\xi^{3} \gamma\|\nabla \omega\|_{L^{2}(\Omega)}^{2}+\xi\left\|\AA^{\frac{1}{2}} \omega\right\|_{L^{2}(\Omega)}^{2} \\
& -\alpha \xi(\nabla \theta, \nabla \omega)_{L^{2}(\Omega)}+\alpha \xi(\nabla \theta, \nabla \omega)_{L^{2}(\Omega)} \\
& +\eta\|\nabla \theta\|_{L^{2}(\Omega)}^{2}+\lambda \eta\|\theta\|_{L^{2}(\Gamma)}^{2}+(\sigma+\beta \xi)\|\theta\|_{L^{2}(\Omega)}^{2}
\end{aligned}
$$

(after noting the cancellation of boundary terms)

$$
\geq C\left[\left\|\AA^{\frac{1}{2}} \omega\right\|_{L^{2}(\Omega)}^{2}+\|\theta\|_{H^{1}(\Omega) \cap L_{\sigma+\lambda}^{2}(\Omega)}^{2}\right]
$$


(where $\langle\cdot, \cdot\rangle$ in $(2.8)$ denotes the pairing between $D\left(\AA^{1 / 2}\right) \times H^{1}(\Omega) \cap L_{\sigma+\lambda}^{2}(\Omega)$ and its dual, and where the constant $C>0$ ). Thus, by Lax-Milgram, $\mathbf{F}^{-1}$ exists as an element of

$$
\mathcal{L}\left(\left[D\left(\AA^{\frac{1}{2}}\right)\right]^{\prime} \times\left[H^{1}(\Omega) \cap L_{\sigma+\lambda}^{2}(\Omega)\right]^{\prime}, D\left(\AA^{\frac{1}{2}}\right) \times H^{1}(\Omega) \cap L_{\sigma+\lambda}^{2}(\Omega)\right),
$$

and the Proposition is proved.

To complete the proof of the maximality of $\mathcal{A}_{\gamma}$, we apply the inverse assured by Proposition 2.1 to both sides of (2.5) to obtain

$$
\left\{\begin{array}{l}
{\left[\begin{array}{l}
\omega_{1} \\
\theta
\end{array}\right] \equiv \mathbf{F}^{-1}\left[\begin{array}{l}
\xi P_{\gamma} f_{2}+\xi^{2} P_{\gamma} f_{1} \\
\beta f_{3}+\alpha A_{D}\left(\mathbf{I}-D \gamma_{0}\right) f_{1}
\end{array}\right],} \\
\omega_{2} \equiv \xi \omega_{1}-f_{1}
\end{array}\right.
$$

and a fortiori, one has, by using the second equation in (2.5), that

$$
A_{R} \theta=-\frac{\beta \xi}{\eta} \theta-\frac{\alpha \xi}{\eta} A_{D}\left(\mathbf{I}-D \gamma_{0}\right) \omega_{1}+\frac{\beta}{\eta} f_{3}+\frac{\alpha}{\eta} A_{D}\left(\mathbf{I}-D \gamma_{0}\right) f_{1} \in L^{2}(\Omega),
$$

viz. $\theta \in D\left(A_{R}\right) \cap L_{\sigma+\lambda}^{2}(\Omega)$. This additional regularity of $\theta$, in conjunction with that implied in the first equation of (2.5) (namely, $\AA \omega_{1}+\alpha \AA G_{1} \gamma_{0} \theta-\alpha \lambda \AA G_{2} \gamma_{0} \theta \in H_{\Gamma_{0}, \gamma}^{-1}(\Omega)$ ) and along with the third equation of (2.4), gives that our constructively acquired solution $\left[\omega_{1}, \omega_{2}, \theta\right]$ to $(2.3)$ is in $D\left(\mathcal{A}_{\gamma}\right)$ as defined in (1.33). Hence, $\mathcal{A}_{\gamma}$ is maximal dissipative and the proof of Theorem 1.1 is complete.

2.2. Proof of Theorem 1.2. By definition, if $\left[\omega^{0}, \omega^{1}, \theta^{0}\right] \in D\left(\mathcal{A}_{\gamma}\right)$, then $\omega^{1} \in$ $D\left(\AA^{1 / 2}\right)$ and $\theta^{0} \in D\left(A_{R}\right)$, and

$$
\AA \omega^{0}+\alpha \AA G_{1} \gamma_{0} \theta^{0}-\alpha \lambda \AA G_{2} \gamma_{0} \theta^{0}=g \in H_{\Gamma_{0}, \gamma}^{-1}(\Omega)=\left[D\left(\AA^{\frac{1}{4}}\right)\right]^{\prime} ;
$$

as $\AA^{-1}:\left[D\left(\AA^{1 / 4}\right)\right]^{\prime} \rightarrow D\left(\AA^{3 / 4}\right) \subset H^{3}(\Omega)$ (this containment deduced by the last characterization in (1.4)), we have after applying $\AA^{-1}$ to (2.10), the use of trace theory and the regularity posted in (1.19) that

$$
\omega^{0}=\AA^{-1} g-\alpha G_{1} \gamma_{0} \theta^{0}+\alpha \lambda G_{2} \gamma_{0} \theta^{0} \in H^{3}(\Omega) .
$$

Thus for $\left[\omega^{0}, \omega^{1}, \theta^{0}\right] \in D\left(\mathcal{A}_{\gamma}^{2}\right)$,

$$
\begin{aligned}
& \mathcal{A}_{\gamma}\left[\begin{array}{l}
\omega^{0} \\
\omega^{1} \\
\theta^{0}
\end{array}\right] \\
& =\left[\begin{array}{c}
P_{\gamma}^{-1}\left[-\AA \omega^{0}-\alpha \AA G_{1} \gamma_{0} \theta^{0}+\alpha \lambda \AA G_{2} \gamma_{0} \theta^{0}+\alpha\left(A_{R} \theta^{0}-\frac{\sigma}{\eta} \theta^{0}\right)\right] \\
-\frac{\eta}{\beta} A_{R} \theta^{0}-\frac{\alpha}{\beta} A_{D}\left(\mathbf{I}-D \gamma_{0}\right) \omega^{1}
\end{array}\right] \in D\left(\mathcal{A}_{\gamma}\right),
\end{aligned}
$$

and (2.12) coupled with (2.11) implies that

$$
\omega^{1} \in H^{3}(\Omega) .
$$


Moreover, (2.12) also has that

$$
P_{\gamma}^{-1}\left[\AA \omega^{0}+\alpha \AA G_{1} \gamma_{0} \theta^{0}-\alpha \lambda \AA G_{2} \gamma_{0} \theta^{0}-\alpha\left(A_{R} \theta^{0}-\frac{\sigma}{\eta} \theta^{0}\right)\right]=g,
$$

where $g \in D\left(\AA^{1 / 2}\right)$, or equivalently

$$
\AA \omega^{0}+\gamma \AA G_{2} \gamma_{1} g+\alpha \AA G_{1} \gamma_{0} \theta^{0}-\alpha \lambda \AA G_{2} \gamma_{0} \theta^{0}=g-\gamma \Delta g-\alpha \Delta \theta^{0} \in L^{2}(\Omega),
$$

after using (1.29). A fortiori then, $\omega^{0}+\gamma G_{2} \gamma_{1} g+\alpha G_{1} \gamma_{0} \theta^{0}-\alpha \lambda G_{2} \gamma_{0} \theta^{0} \in D(\AA) \subset$ $H^{4}(\Omega)$. But trace theory and the smoothing specified in (1.19) give that $G_{2} \gamma_{1} g, G_{1} \gamma_{0} \theta^{0}$ and $G_{2} \gamma_{0} \theta^{0} \in H^{4}(\Omega)$, and thus $D\left(\mathcal{A}_{\gamma}^{2}\right) \subset H^{4}(\Omega) \times H^{3}(\Omega) \times H^{2}(\Omega)$ with the inclusion being continuous. The solution $\left[\omega, \omega_{t}, \theta\right]$ will consequently have the asserted regularity upon consideration of the fundamental property that for $\xi \geq 0,\left[\omega^{0}, \omega^{1}, \theta^{0}\right]$ $\in D\left(\mathcal{A}_{\gamma}^{\xi}\right) \Rightarrow$

$$
\left[\begin{array}{l}
\omega \\
\omega_{t} \\
\theta
\end{array}\right]=e^{\mathcal{A}_{\gamma}(\cdot)}\left[\begin{array}{c}
\omega^{0} \\
\omega^{1} \\
\theta^{0}
\end{array}\right] \in C\left([0, T] ; D\left(\mathcal{A}_{\gamma}^{\xi}\right)\right) .
$$

To prove (ii), we note that with $\left[\omega^{0}, \omega^{1}, \theta^{0}\right] \in D\left(\mathcal{A}_{\gamma}^{2}\right), \omega_{t t} \in C\left([0, T] ; D\left(\AA^{1 / 2}\right)\right)$, so the solution $\left[\omega, \omega_{t}, \theta\right]$ to (1.1) satisfies

$$
-\AA \hat{\omega} \omega+\gamma \AA G_{2} \gamma_{1} \omega_{t t}-\alpha \AA G_{1} \gamma_{0} \theta+\alpha \lambda \AA G_{2} \gamma_{0} \theta=\omega_{t t}-\gamma \Delta \omega_{t t}+\alpha \Delta \theta
$$

in $C\left([0, T] ; L^{2}(\Omega)\right)$, which establishes the result.

REMARK 3. Because of the regularity result posted in Theorem 1.2 (ii), we have for sufficiently smooth initial data the valid pointwise representation

$$
\omega_{t t}+\Delta^{2} \omega-\gamma \Delta \omega_{t t}+\alpha \Delta \theta=0 .
$$

REMARK 4. If either $\lambda$ or $\sigma>0$, then for initial data $\left[\omega^{0}, \omega^{1}, \theta^{0}\right] \in D\left(\mathcal{A}_{\gamma}^{2}\right)$, we will also have that the solution component $\theta$ of 1.1 is in $C\left([0, T] ; H^{3}(\Omega)\right)$. In fact, the last component on the right-hand side of (2.12), the definition of $D\left(\mathcal{A}_{\gamma}\right)$, and (2.13) give that

$$
A_{R} \theta^{0}=h+\frac{\alpha}{\eta} \Delta \omega^{1} \in H^{1}(\Omega),
$$

where $h \in H^{2}(\Omega)$. Applying $A_{R}^{-1}$ (which exists for $\lambda$ or $\sigma>0$ ) to both sides of (2.19) thus yields

$$
\theta^{0} \in H^{3}(\Omega)
$$

and the result will follow from the semigroup property posted in (2.16).

2.3. Proof of Theorem 1.3. In proving Theorem 1.3, we begin with a preliminary energy identity.

LEMMA 2.2. Again, with initial data $\left[\omega^{0}, \omega^{1}, \theta^{0}\right] \in \boldsymbol{H}_{\gamma}$, we have that the component $\theta$ of the solution of $(1.1)$ is an element of $L^{2}\left(0, \infty ; H^{1}(\Omega) \cap L_{\sigma+\lambda}^{2}(\Omega)\right)$; indeed, we have the following relation $\forall T>0$ :

$$
-2 \eta \int_{0}^{T}\|\theta\|_{H^{1}(\Omega) \cap L_{\sigma+\lambda}^{2}(\Omega)}^{2} d t=E_{\gamma}(T)-E_{\gamma}(0),
$$


where the "energy" $E_{\gamma}(t)$ is defined by

$$
E_{\gamma}(t) \equiv\left\|\dot{A}^{\frac{1}{2}} \omega(t)\right\|_{L^{2}(\Omega)}^{2}+\left\|P_{\gamma}^{\frac{1}{2}} \omega_{t}(t)\right\|_{L^{2}(\Omega)}^{2}+\|\theta\|_{L_{\sigma+\lambda}^{2}(\Omega)}^{2},
$$

and where the norm of $H^{1}(\Omega) \cap L_{\sigma+\lambda}^{2}(\Omega)$ is as defined in (1.14).

Proof. Starting with initial data in $D\left(\mathcal{A}_{\gamma}\right)$ which will provide $\forall T>0$ that the solution $\left[\omega, \omega_{t}, \theta\right] \in C\left([0, T] ; D\left(\mathcal{A}_{\gamma}\right)\right)$ and $\left[\omega_{t}, \omega_{t t}, \theta_{t}\right] \in C\left([0, T] ; \mathbf{H}_{\gamma}\right)$, we have pointwise on $(0, T)$

$$
\frac{d}{d t}\left\|\left[\begin{array}{c}
\omega(t) \\
\omega_{t}(t) \\
\theta(t)
\end{array}\right]\right\|_{\mathbf{H}_{\gamma}}^{2}=2\left(\mathcal{A}_{\gamma}\left[\begin{array}{c}
\omega(t) \\
\omega_{t}(t) \\
\theta(t)
\end{array}\right],\left[\begin{array}{c}
\omega(t) \\
\omega_{t}(t) \\
\theta(t)
\end{array}\right]\right)_{\mathbf{H}_{\gamma}},
$$

and for this special choice of initial data we will have the desired equality (2.21) upon integration and using the fact from (1.12) that

$$
\begin{aligned}
& \left(A_{R} \theta, \theta\right)_{L^{2}(\Omega)}=\left(-\Delta \theta+\frac{\sigma}{\eta} \theta, \theta\right)_{L^{2}(\Omega)} \\
& =\|\nabla \theta\|_{L^{2}(\Omega)}^{2}+\frac{\sigma}{\eta}\|\theta\|_{L^{2}(\Omega)}^{2}+\lambda\|\theta\|_{L^{2}(\Gamma)}^{2} \text { for } \theta \in D\left(A_{R}\right) .
\end{aligned}
$$

The asserted $L^{2}$-regularity follows immediately from (2.21), using the norm definition (1.14) for $H^{1}(\Omega) \cap L_{\sigma+\lambda}^{2}(\Omega)$, and the fact that $\left\{e^{\mathcal{A}_{\gamma} t}\right\}_{t \geq 0}$ is a contraction semigroup. A density argument concludes the proof.

REMARK 5. J. Lagnese in [12] first showed the dissipativity property (2.21) through a formal integration and a subsequent justification through variational arguments, and the alternate proof is included here as a simple consequence of contractive semigroups.

We next derive a trace regularity result for the model under consideration here, a regularity which does not follow from the standard Sobolev trace theory, and which is critical in our estimates of uniform decay. We note that related trace regularity results for Euler-Bernoulli plates were proved in [18] and for Kirchoff plates in [14].

LEMMA 2.3. One has the component $\omega$ of the solution $\left[\omega, \omega_{t}, \theta\right]$ of (1.1) satisfies $\left.\Delta \omega\right|_{\Gamma_{0}} \in L^{2}\left(0, T ; L^{2}\left(\Gamma_{0}\right)\right)$ with the estimate

$$
\begin{aligned}
\int_{0}^{T}\|\Delta \omega\|_{L^{2}\left(\Gamma_{0}\right)}^{2} d t \leq C\left(\int _ { 0 } ^ { T } \left[\left\|\AA^{\frac{1}{2}} \omega\right\|_{L^{2}(\Omega)}^{2}\right.\right. & \left.+\left\|P_{\gamma}^{\frac{1}{2}} \omega_{t}\right\|_{L^{2}(\Omega)}^{2}+\|\theta\|_{H^{1}(\Omega) \cap L_{\sigma+\lambda}^{2}(\Omega)}^{2}\right] d t \\
& \left.+E_{\gamma}(T)+E_{\gamma}(0)\right)
\end{aligned}
$$

where $C$ does not depend on the parameter $\gamma$.

Proof. If we take initial data $\left[\omega^{0}, \omega^{1}, \theta^{0}\right]$ in $D\left(\mathcal{A}_{\gamma}^{2}\right)$, then Theorem 1.2 provides that $\left[\omega, \omega_{t}, \theta\right]$ is a classical pointwise solution of (1.1). We will work to extract the desired estimate (2.24) in this special case - and consequently for all initial data after an extension by continuity - by multiplying the first equation of (1.1) by the quantity $h \cdot \nabla \omega$, where $h(x, y) \equiv\left[h_{1}(x, y), h_{2}(x, y)\right]$ is a $\left[C^{2}(\bar{\Omega})\right]^{2}$ vector field ${ }^{1}$ which satisfies

$$
\left.h\right|_{\Gamma}= \begin{cases}{\left[\nu_{1}, \nu_{2}\right]} & \text { on } \Gamma_{0}, \\ 0 & \text { on } \Gamma_{1},\end{cases}
$$

\footnotetext{
${ }^{1}$ Here is where we use the fact that $\Gamma_{0}$ and $\Gamma_{1}$ are separated.
} 
followed by an integration from 0 to $T$; i.e., we will work with the equation

$$
\int_{0}^{T}\left(\omega_{t t}-\gamma \Delta \omega_{t t}+\Delta^{2} \omega+\alpha \Delta \theta, h \cdot \nabla \omega\right)_{L^{2}(\Omega)} d t=0 .
$$

(i) First,

$$
\begin{aligned}
& \int_{0}^{T}\left(\omega_{t t}, h \cdot \nabla \omega\right)_{L^{2}(\Omega)} d t=\left.\left(\omega_{t}, h \cdot \nabla \omega\right)_{L^{2}(\Omega)}\right|_{0} ^{T}-\int_{0}^{T}\left(\omega_{t}, h \cdot \nabla \omega_{t}\right)_{L^{2}(\Omega)} d t \\
= & \left.\left(\omega_{t}, h \cdot \nabla \omega\right)_{L^{2}(\Omega)}\right|_{0} ^{T}-\frac{1}{2} \int_{0}^{T} \int_{\Omega} \operatorname{div}\left(\omega_{t}^{2} h\right) d t d \Omega \\
& +\frac{1}{2} \int_{0}^{T} \int_{\Omega} \omega_{t}^{2}\left[h_{1 x}+h_{2 y}\right] d t d \Omega \\
= & \left.\left(\omega_{t}, h \cdot \nabla \omega\right)_{L^{2}(\Omega)}\right|_{0} ^{T}+\frac{1}{2} \int_{0}^{T} \int_{\Omega} \omega_{t}^{2}\left[h_{1 x}+h_{2 y}\right] d t d \Omega,
\end{aligned}
$$

after making use of the divergence theorem and the fact that $\omega_{t}=0$ on $\Gamma_{0}$.

(ii) Next,

$$
\begin{aligned}
& \int_{0}^{T}\left(-\Delta \omega_{t t}, h \cdot \nabla \omega\right)_{L^{2}(\Omega)} d t \\
& =\left.\left(\nabla \omega_{t}, \nabla(h \cdot \nabla \omega)\right)_{L^{2}(\Omega)}\right|_{0} ^{T}-\int_{0}^{T}\left(\nabla \omega_{t}, \nabla\left(h \cdot \nabla \omega_{t}\right)\right)_{L^{2}(\Omega)} d t \\
& =\left.\left(\nabla \omega_{t}, \nabla(h \cdot \nabla \omega)\right)_{L^{2}(\Omega)}\right|_{0} ^{T}-\frac{1}{2} \int_{0}^{T} \int_{\Omega} \operatorname{div}\left(\left|\nabla \omega_{t}\right|^{2} h\right) d t d \Omega \\
& -\int_{0}^{T} \int_{\Omega}\left[\frac{\omega_{t x}^{2} h_{1 x}}{2}+\frac{\omega_{t y}^{2} h_{2 y}}{2}\right] d t d \Omega-\int_{0}^{T} \int_{\Omega}\left[\omega_{t x} \omega_{t y} h_{2 x}+\omega_{t x} \omega_{t y} h_{1 y}\right] d t d \Omega \\
& +\int_{0}^{T} \int_{\Omega}\left[\frac{\omega_{t x}^{2} h_{2 y}}{2}+\frac{\omega_{t y}^{2} h_{1 x}}{2}\right] d t d \Omega \\
& \left.=\left.\left(\nabla \omega_{t}, h \cdot \nabla \omega\right)_{L^{2}(\Omega)}\right|_{0} ^{T}\right] \\
& +\int_{0}^{T} \int_{\Omega}\left[\frac{\omega_{t x}^{2} h_{2 y}}{2}+\frac{\omega_{t y}^{2} h_{1 x}}{2}-\frac{\omega_{t x}^{2} h_{1 x}}{2}-\frac{\omega_{t y}^{2} h_{2 y}}{2}\right] d t d \Omega \\
& -\int_{0}^{T} \int_{\Omega}\left[\omega_{t x} \omega_{t y} h_{2 x}+\omega_{t x} \omega_{t y} h_{1 y}\right] d t d \Omega,
\end{aligned}
$$

after again using the divergence theorem and the fact that

$$
\int_{\Omega} \operatorname{div}\left(\left|\nabla \omega_{t}\right|^{2} h\right) d \Omega=\int_{\Gamma_{0}}\left|\nabla \omega_{t}\right|^{2} d \Gamma_{0}=0\left(\text { as } \omega_{t}(t) \in H_{\Gamma_{0}}^{2}(\Omega)\right) .
$$


(iii) To handle the fourth-order term, we use Green's theorem (1.5), the given boundary conditions of $(1.1),(2.25)$, and the fact that $\omega \in H_{\Gamma_{0}}^{2}(\Omega)$ to obtain

$$
\begin{aligned}
& \int_{0}^{T}\left(\Delta^{2} \omega, h \cdot \nabla \omega\right)_{L^{2}(\Omega)} d t=\int_{0}^{T} a(\omega, h \cdot \nabla \omega) d t \\
& +\alpha \int_{0}^{T} \int_{\Gamma_{1}} \theta \cdot \frac{\partial h \cdot \nabla \omega}{\partial \nu} d \Gamma_{1} d t-\int_{0}^{T} \int_{\Gamma_{0}}\left(\Delta \omega+(1-\mu) B_{1} \omega\right) \frac{\partial^{2} \omega}{\partial \nu^{2}} d \Gamma_{0} d t .
\end{aligned}
$$

We note at this point that we can rewrite the first term on the right-hand side of $(2.29)$ as

$$
\begin{array}{rl}
\int_{0}^{T} & a(\omega, h \cdot \nabla \omega) d t=\frac{1}{2} \int_{0}^{T} \int_{\Omega} h \cdot \nabla\left[\omega_{x x}^{2}+\omega_{y y}^{2}+2 \mu \omega_{x x} \omega_{y y}+2(1-\mu) \omega_{x y}^{2}\right] d t d \Omega \\
& +\mathcal{O}\left(\int_{0}^{T}\left\|\AA^{\frac{1}{2}} \omega\right\|_{L^{2}(\Omega)}^{2} d t\right),
\end{array}
$$

where $\mathcal{O}\left(\int_{0}^{T}\left\|\AA^{1 / 2} \omega\right\|_{L^{2}(\Omega)}^{2} d t\right)$ denotes a series of terms which can be majorized by the $L^{2}\left(0, T ; D\left(\AA^{1 / 2}\right)\right)$-norm of $\omega$; we consequently have by the divergence theorem that

$$
\begin{aligned}
& \int_{0}^{T} a(\omega, h \cdot \nabla \omega) d t= \\
& \frac{1}{2} \int_{0}^{T} \int_{\Omega} h \cdot \nabla\left[\omega_{x x}^{2}+\omega_{y y}^{2}+2 \mu \omega_{x x} \omega_{y y}+2(1-\mu) \omega_{x y}^{2}\right] d t d \Omega \\
& +\mathcal{O}\left(\int_{0}^{T}\left\|\AA^{\frac{1}{2}} \omega\right\|_{L^{2}(\Omega)}^{2} d t\right) \\
= & \frac{1}{2} \int_{0}^{T} \int_{\Omega} \operatorname{div}\left\{h\left[\omega_{x x}^{2}+\omega_{y y}^{2}+2 \mu \omega_{x x} \omega_{y y}+2(1-\mu) \omega_{x y}^{2}\right]\right\} \\
& +\mathcal{O}\left(\int_{0}^{T}\left\|\AA^{\frac{1}{2}} \omega\right\|_{L^{2}(\Omega)}^{2} d t\right) \\
= & \frac{1}{2} \int_{0}^{T} \int_{\Gamma_{0}}\left[\omega_{x x}^{2}+\omega_{y y}^{2}+2 \mu \omega_{x x} \omega_{y y}+2(1-\mu) \omega_{x y}^{2}\right] d t d \Gamma_{0} \\
& +\mathcal{O}\left(\int_{0}^{T}\left\|\AA^{\frac{1}{2}} \omega\right\|_{L^{2}(\Omega)}^{2} d t\right) \\
= & \frac{1}{2} \int_{0}^{T} \int_{\Gamma_{0}}(\Delta \omega)^{2} d t+\mathcal{O}\left(\int_{0}^{T}\left\|\AA^{\frac{1}{2}} \omega\right\|_{L^{2}(\Omega)}^{2} d t\right),
\end{aligned}
$$

where in the last step above, we have used the fact (as reasoned in [12, Ch. 4] that $\left.\omega\right|_{\Gamma_{0}}=\left.\frac{\partial \omega}{\partial \nu}\right|_{\Gamma_{0}}=0$ implies that $\omega_{x x}^{2}+\omega_{y y}^{2}+2 \mu \omega_{x x} \omega_{y y}+2(1-\mu) \omega_{x y}^{2}=$ $(\Delta \omega)^{2}$ on $\Gamma_{0}$. 
To handle the last term on the right-hand side of (2.29), we note that $B_{1} \omega=0$ on $\Gamma_{0}$, which implies that

$$
\Delta \omega=\Delta \omega+(1-\mu) B_{1} \omega=\frac{\partial^{2} \omega}{\partial \nu^{2}} \text { on } \Gamma_{0} ;
$$

we consequently have upon the insertion of (2.31) into (2.29), as well as by the consideration of (2.32), that

$$
\begin{aligned}
& \int_{0}^{T}\left(\Delta^{2} \omega, h \cdot \nabla \omega\right)_{L^{2}(\Omega)} d t=-\frac{1}{2} \int_{0}^{T}\|\Delta \omega\|_{L^{2}\left(\Gamma_{0}\right)}^{2} d t \\
& +\alpha \int_{0}^{T} \int_{\Gamma_{1}} \theta \cdot \frac{\partial h \cdot \nabla \omega}{\partial \nu} d \Gamma_{1} d t+\mathcal{O}\left(\int_{0}^{T}\left\|\AA^{\frac{1}{2}} \omega\right\|_{L^{2}(\Omega)}^{2} d t\right) .
\end{aligned}
$$

(iv) To handle the last term on the left-hand side of equation (2.26), we again use Green's theorem and the boundary conditions posted in (1.1) to obtain

$$
\int_{0}^{T}(\Delta \theta, h \cdot \nabla \omega)_{L^{2}(\Omega)} d t=-\int_{0}^{T}(\nabla \theta, \nabla(h \cdot \nabla \omega))_{L^{2}(\Omega)} d t .
$$

To finish the proof, we rewrite (2.26) by collecting the relations given above in (2.27), (2.28), (2.33), and (2.34) to attain the desired inequality (2.24), upon the taking of norms and a subsequent majorization.

In showing the exponential decay of the semigroup $\left\{e^{\mathcal{A}_{\gamma} t}\right\}_{t \geq 0}$ (Theorem 1.3) it will suffice as usual, to prove that there exists a time $0<T<\infty$ which satisfies for all initial data in $\mathbf{H}_{\gamma}$,

$$
E_{\gamma}(T) \leq \xi E_{\gamma}(0) \text { with } \xi<1 .
$$

By a density argument, it will then be enough by Lemma 2.2 to show the existence of a time $T, 0<T<\infty$, and a positive constant $C_{T}$ (independent of $\gamma$ ) for initial data in $\left[\omega^{0}, \omega^{1}, \theta^{0}\right] \in D\left(\mathcal{A}_{\gamma}^{2}\right)$ such that

$$
E_{\gamma}(T) \leq C_{T} \int_{0}^{T}\|\theta\|_{H^{1}(\Omega) \cap L_{\sigma+\lambda}^{2}(\Omega)}^{2} d t
$$

to which end we will proceed to work.

2.4. Proof of inequality (2.36). Because of Theorem 1.2, we have for initial data $\left[\omega^{0}, \omega^{1}, \theta^{0}\right] \in D\left(\mathcal{A}_{\gamma}^{2}\right)$ a classical pointwise solution $\left[\omega, \omega_{t}, \theta\right]$ of $(1.1)$; we can thus multiply the first equation in (1.1) by $A_{D}^{-1} \theta$ and integrate in time and space to obtain

$$
\int_{0}^{T}\left(\omega_{t t}-\gamma \Delta \omega_{t t}+\Delta^{2} \omega+\alpha \Delta \theta, A_{D}^{-1} \theta\right)_{L^{2}(\Omega)} d t=0
$$

the bulk of the work from here on out will be the scrutiny of the left-hand side of this equation.

(A.1) Dealing with $\int_{0}^{T}\left(\omega_{t t}-\gamma \Delta \omega_{t t}, A_{D}^{-1} \theta\right)_{L^{2}(\Omega)} d t$. Using an integration by parts, the second differential equation of (1.1) and the fact that $A_{R} \theta=-\Delta \theta+\frac{\sigma}{\eta} \theta=-\Delta \theta+$ 
$\Delta D \gamma_{0} \theta+\frac{\sigma}{\eta} \theta=A_{D}\left(\mathbf{I}-D \gamma_{0}\right) \theta+\frac{\sigma}{\eta} \theta$ produce

$$
\begin{aligned}
& \int_{0}^{T}\left(\omega_{t t}-\gamma \Delta \omega_{t t}, A_{D}^{-1} \theta\right)_{L^{2}(\Omega)} d t \\
= & \left.\left(\omega_{t}, A_{D}^{-1} \theta\right)_{L^{2}(\Omega)}\right|_{0} ^{T}+\left.\gamma\left(\nabla \omega_{t}, \nabla A_{D}^{-1} \theta\right)_{L^{2}(\Omega)}\right|_{0} ^{T} \\
& -\int_{0}^{T}\left[\left(\omega_{t}, A_{D}^{-1} \theta_{t}\right)_{L^{2}(\Omega)}+\gamma\left(\nabla \omega_{t}, \nabla A_{D}^{-1} \theta_{t}\right)_{L^{2}(\Omega)}\right] d t \\
= & \alpha \beta^{-1} \int_{0}^{T}\left[\left\|\omega_{t}\right\|_{L^{2}(\Omega)}^{2}+\gamma\left\|\nabla \omega_{t}\right\|_{L^{2}(\Omega)}^{2}\right] d t \\
& -\alpha \beta^{-1} \int_{0}^{T}\left[\left(\omega_{t}, D \gamma_{0} \omega_{t}\right)_{L^{2}(\Omega)}+\gamma\left(\nabla \omega_{t}, \nabla D \gamma_{0} \omega_{t}\right)_{L^{2}(\Omega)}\right] d t \\
& +\eta \beta^{-1} \int_{0}^{T}\left[\left(\omega_{t},\left(\mathbf{I}-D \gamma_{0}\right) \theta\right)_{L^{2}(\Omega)}+\gamma\left(\nabla \omega_{t}, \nabla\left(\mathbf{I}-D \gamma_{0}\right) \theta\right)_{L^{2}(\Omega)}\right] d t \\
& +\sigma \beta^{-1} \int_{0}^{T}\left[\left(\omega_{t}, A_{D}^{-1} \theta\right)_{L^{2}(\Omega)}+\gamma\left(\nabla \omega_{t}, \nabla A_{D}^{-1} \theta\right)_{L^{2}(\Omega)}\right] d t \\
& +\left.\left(\omega_{t}, A_{D}^{-1} \theta\right)_{L^{2}(\Omega)}\right|_{0} ^{T}+\left.\gamma\left(\nabla \omega_{t}, \nabla A_{D}^{-1} \theta\right)_{L^{2}(\Omega)}\right|_{0} ^{T} .
\end{aligned}
$$

A further integration by parts, an application of Green's theorem (1.5) to the term $\int_{0}^{T}\left(\nabla \omega_{t}, \nabla D \gamma_{0} \omega_{t}\right)_{L^{2}(\Omega)} d t$, and a consideration of the boundary conditions posted in (1.1) yield

$$
\begin{aligned}
& -\gamma \int_{0}^{T}\left(\nabla \omega_{t}, \nabla D \gamma_{0} \omega_{t}\right)_{L^{2}(\Omega)} d t \\
= & -\left.\gamma\left(\nabla \omega_{t}, \nabla D \gamma_{0} \omega\right)_{L^{2}(\Omega)}\right|_{0} ^{T}+\gamma \int_{0}^{T}\left(\nabla \omega_{t t}, \nabla D \gamma_{0} \omega\right)_{L^{2}(\Omega)} d t \\
= & -\left.\gamma\left(\nabla \omega_{t}, \nabla D \gamma_{0} \omega\right)_{L^{2}(\Omega)}\right|_{0} ^{T}-\gamma \int_{0}^{T}\left(\Delta \omega_{t t}, D \gamma_{0} \omega\right)_{L^{2}(\Omega)} d t \\
& +\gamma \int_{0}^{T}\left(\frac{\partial \omega_{t t}}{\partial \nu}, \gamma_{0} \omega\right)_{L^{2}\left(\Gamma_{1}\right)} d t \\
= & -\left.\gamma\left(\nabla \omega_{t}, \nabla D \gamma_{0} \omega\right)_{L^{2}(\Omega)}\right|_{0} ^{T}-\int_{0}^{T}\left(\omega_{t t}+\Delta^{2} \omega+\alpha \Delta \theta, D \gamma_{0} \omega\right)_{L^{2}(\Omega)} d t \\
& +\gamma \int_{0}^{T}\left(\frac{\partial \omega_{t t}}{\partial \nu}, \gamma_{0} \omega\right)_{L^{2}\left(\Gamma_{1}\right)} d t \\
= & -\left.\gamma\left(\nabla \omega_{t}, \nabla D \gamma_{0} \omega\right)_{L^{2}(\Omega)}\right|_{0} ^{T}-\left.\left(\omega_{t}, D \gamma_{0} \omega\right)_{L^{2}(\Omega)}\right|_{0} ^{T}+\int_{0}^{T}\left(\omega_{t}, D \gamma_{0} \omega_{t}\right)_{L^{2}(\Omega)} d t \\
& -\int_{0}^{T} a\left(D \gamma_{0} \omega, \omega\right) d t-\int_{0}^{T}\left(\alpha \theta, \frac{\partial D \gamma_{0} \omega}{\partial \nu}\right)_{L^{2}\left(\Gamma_{1}\right)} d t-\int_{0}^{T}\left(\Delta \omega, \frac{\partial D \gamma_{0} \omega}{\partial \nu}\right)_{L^{2}\left(\Gamma_{0}\right)} d t \\
& +\alpha \int_{0}^{T}\left(\nabla \theta, \nabla D \gamma_{0} \omega\right)_{L^{2}(\Omega)} d t .
\end{aligned}
$$


Given that $D \gamma_{0} \in \mathcal{L}\left(H^{s}(\Omega)\right)$ for all real $s$ and further using the fact that $A_{D}^{-1}$ is "smoothing," viz. $\left\|A_{D}^{-1} \theta\right\|_{H^{2}(\Omega)} \leq C\|\theta\|_{L^{2}(\Omega)}$, we have the following estimates for the solution $\left[\omega, \omega_{t}, \theta\right]$ of (1.1) corresponding to arbitrary initial data in $\mathbf{H}_{\gamma}$ :

$$
\begin{aligned}
& \left\|\left(I-D \gamma_{0}\right) \theta\right\|_{L^{2}(\Omega)}+\left\|A_{D}^{-1} \theta\right\|_{L^{2}(\Omega)} \leq C\|\theta\|_{H^{1}(\Omega) \cap L_{\sigma+\lambda}^{2}(\Omega)} ; \\
& \left\|\nabla\left(I-D \gamma_{0}\right) \theta\right\|_{L^{2}(\Omega)}+\left\|\nabla A_{D}^{-1} \theta\right\|_{L^{2}(\Omega)} \leq C\|\theta\|_{H^{1}(\Omega) \cap L_{\sigma+\lambda}^{2}(\Omega)} ; \\
& \left\|\nabla D \gamma_{0} \omega\right\|_{L^{2}(\Omega)} \leq C\left\|\AA^{\frac{1}{2}} \omega\right\|_{L^{2}(\Omega)} ; \\
& \left\|\frac{\partial D \gamma_{0} \omega}{\partial \nu}\right\|_{H^{\frac{1}{2}(\Gamma)}} \leq C\left\|\AA^{\frac{1}{2}} \omega\right\|_{L^{2}(\Omega)} .
\end{aligned}
$$

Thus a substitution of (2.39) into (2.38) and a subsequent majorization which makes use of the inequalities (2.40)-(2.43) will give the estimate

$$
\begin{aligned}
& \quad\left|\int_{0}^{T}\left(\omega_{t t}-\gamma \Delta \omega_{t t}, A_{D}^{-1} \theta\right)_{L^{2}(\Omega)} d t-\alpha \beta^{-1} \int_{0}^{T}\left[\left\|\omega_{t}\right\|_{L^{2}(\Omega)}^{2}+\gamma\left\|\nabla \omega_{t}\right\|_{L^{2}(\Omega)}^{2}\right] d t\right| \\
& \leq C \int_{0}^{T}\left[\left\|\omega_{t}\right\|_{L^{2}(\Omega)}\|\theta\|_{H^{1}(\Omega) \cap L_{\sigma+\lambda}^{2}(\Omega)}+\gamma\left\|\nabla \omega_{t}\right\|_{L^{2}(\Omega)}\|\theta\|_{H^{1}(\Omega) \cap L_{\sigma+\lambda}^{2}(\Omega)}\right] d t \\
& +C\left[E_{\gamma}(0)+E_{\gamma}(T)\right]+\left|\int_{0}^{T} a\left(D \gamma_{0} \omega, \omega\right) d t\right| \\
& +\left|\int_{0}^{T}\left(\Delta \omega, \frac{\partial D \gamma_{0} \omega}{\partial \nu}\right)_{L^{2}\left(\Gamma_{0}\right)} d t\right| \\
& \leq \epsilon \int_{0}^{T}\left[\left\|\AA^{\frac{1}{2}} \omega\right\|_{L^{2}(\Omega)}^{2}+\left\|\omega_{t}\right\|_{L^{2}(\Omega)}^{2}+\gamma\left\|\nabla \omega_{t}\right\|_{L^{2}(\Omega)}^{2}\right] d t+C_{\epsilon} \int_{0}^{T}\|\theta\|_{H^{1}(\Omega) \cap L_{\sigma+\lambda}^{2}(\Omega)}^{2} d t \\
& +C\left[E_{\gamma}(0)+E_{\gamma}(T)\right]+\frac{\alpha}{\beta}\left|\int_{0}^{T} a\left(D \gamma_{0} \omega, \omega\right) d t\right| \\
& +\frac{\alpha}{\beta}\left|\int_{0}^{T}\left(\Delta \omega, \frac{\partial D \gamma_{0} \omega}{\partial \nu}\right)_{L^{2}\left(\Gamma_{0}\right)} d t\right|,
\end{aligned}
$$

where the constants $C$ and $C_{\epsilon}$ do not depend on $\gamma, 0<\gamma \leq M$.

(A.2) Dealing with $\int_{0}^{T}\left(\Delta^{2} \omega, A_{D}^{-1} \theta\right) d t$. Yet another application of Green's theorem in (1.5) and the use of the enforced boundary conditions in (1.1) give

$$
\begin{gathered}
\int_{0}^{T}\left(\Delta^{2} \omega, A_{D}^{-1} \theta\right) d t=\int_{0}^{T} a\left(\omega, A_{D}^{-1} \theta\right) d t-\int_{0}^{T}\left(\Delta \omega, \frac{\partial A_{D}^{-1} \theta}{\partial \nu}\right)_{L^{2}\left(\Gamma_{0}\right)} d t \\
(2.45)+\alpha \int_{0}^{T}\left(\theta, \frac{\partial A_{D}^{-1} \theta}{\partial \nu}\right)_{L^{2}\left(\Gamma_{1}\right)} d t .
\end{gathered}
$$


Estimating the right-hand side of (2.45) yields, after the use of trace theory, elliptic regularity and the mean inequality,

$$
\begin{aligned}
& \left|\int_{0}^{T}\left(\Delta^{2} \omega, A_{D}^{-1} \theta\right) d t\right| \\
\leq & C_{0} \int_{0}^{T}\left\|\AA^{\frac{1}{2}} \omega\right\|_{L^{2}(\Omega)}\|\theta\|_{H^{1}(\Omega) \cap L_{\sigma+\lambda}^{2}(\Omega)} d t \\
& +\frac{\epsilon}{2 C} \int_{0}^{T}\|\Delta \omega\|_{L^{2}\left(\Gamma_{0}\right)}^{2} d t+C_{\epsilon} \int_{0}^{T}\|\theta\|_{H^{1}(\Omega) \cap L_{\sigma+\lambda}^{2}(\Omega)}^{2} d t
\end{aligned}
$$

(where the inverted $C$ is the same constant present in (2.24))

$$
\begin{aligned}
\leq & C_{0} \int_{0}^{T}\left\|\AA^{\frac{1}{2}} \omega\right\|_{L^{2}(\Omega)}\|\theta\|_{H^{1}(\Omega) \cap L_{\sigma+\lambda}^{2}(\Omega)} d t \\
& +\frac{\epsilon}{2}\left[\int_{0}^{T}\left(\left\|\AA^{\frac{1}{2}} \omega\right\|_{L^{2}(\Omega)}^{2}+\left\|P_{\gamma}^{\frac{1}{2}} \omega_{t}\right\|_{L^{2}(\Omega)}^{2}\right) d t+\right. \\
& \left.+E_{\gamma}(0)+E_{\gamma}(T)\right]+C_{\epsilon} \int_{0}^{T}\|\theta\|_{H^{1}(\Omega) \cap L_{\sigma+\lambda}^{2}(\Omega)}^{2} d t
\end{aligned}
$$

(by Lemma 2.3)

$$
\begin{aligned}
\leq & \epsilon \int_{0}^{T}\left[\left\|\AA^{\frac{1}{2}} \omega\right\|_{L^{2}(\Omega)}^{2}+\left\|P_{\gamma}^{\frac{1}{2}} \omega_{t}\right\|_{L^{2}(\Omega)}^{2}\right] d t \\
& +C\left[E_{\gamma}(0)+E_{\gamma}(T)\right]+C_{\epsilon} \int_{0}^{T}\|\theta\|_{H^{1}(\Omega) \cap L_{\sigma+\lambda}^{2}(\Omega)}^{2} d t,
\end{aligned}
$$

after the use of the mean inequality.

(A.3) Dealing with $\int_{0}^{T}\left(\alpha \Delta \theta, A_{D}^{-1} \theta\right)_{L^{2}(\Omega)} d t$. Finally, for the last term of (2.37), again using the fact that $A_{R} \theta=A_{D}\left(\mathbf{I}-D \gamma_{0}\right) \theta+\frac{\sigma}{\eta} \theta$, we have easily

$$
\begin{aligned}
\alpha \int_{0}^{T} & \left(A_{D}\left(\mathbf{I}-D \gamma_{0}\right) \theta+\frac{\sigma}{\eta} \theta, A_{D}^{-1} \theta\right)_{L^{2}(\Omega)} d t \\
\quad & \alpha \int_{0}^{T}\left[\|\theta\|_{L^{2}(\Omega)}^{2}-\left(D \gamma_{0} \theta, \theta\right)_{L^{2}(\Omega)}+\left(\frac{\alpha \sigma}{\eta} A_{D}^{-1} \theta, \theta\right)_{L^{2}(\Omega)}\right] d t \\
& \leq C \int_{0}^{T}\|\theta\|_{H^{1}(\Omega) \cap L_{\sigma+\lambda}^{2}(\Omega)}^{2} .
\end{aligned}
$$

(A.4) Combining (2.37), (2.44), (2.46), and (2.47) thus results in the following. For $\epsilon>0$ small enough there exists a constant $C>0$ (independent of $\gamma$ ) such that the solution $\left[\omega, \omega_{t}, \theta\right]$ of $(1.1)$ satisfies

$$
\begin{aligned}
& \left(\frac{\alpha}{\beta}-2 \epsilon\right) \int_{0}^{T}\left[\left\|\omega_{t}\right\|_{L^{2}(\Omega)}^{2}+\gamma\left\|\nabla \omega_{t}\right\|_{L^{2}(\Omega)}^{2}\right] d t \\
\leq & C\left[\int_{0}^{T}\|\theta\|_{H^{1}(\Omega) \cap L_{\bar{\sigma}+\lambda}^{2}(\Omega)}^{2} d t+E_{\gamma}(T)+E_{\gamma}(0)\right] \\
& +2 \epsilon \int_{0}^{T}\left\|\AA^{\frac{1}{2}} \omega\right\|_{L^{2}(\Omega)}^{2} d t+\frac{\alpha}{\beta}\left|\int_{0}^{T} a\left(D \gamma_{0} \omega, \omega\right) d t\right| \\
& +\frac{\alpha}{\beta}\left|\int_{0}^{T}\left(\Delta \omega, \frac{\partial D \gamma_{0} \omega}{\partial \nu}\right)_{L^{2}\left(\Gamma_{0}\right)} d t\right|,
\end{aligned}
$$

where the noncrucial dependence of $C$ upon $\epsilon$ has not been noted. 
(A.5) Estimating the residual terms $\left|\int_{0}^{T} a\left(D \gamma_{0} \omega, \omega\right) d t\right|$ and $\left|\int_{0}^{T}\left(\Delta \omega, \frac{\partial D \gamma_{0} \omega}{\partial \nu}\right)_{L^{2}\left(\Gamma_{0}\right)} d t\right|{ }^{2}$ At this point we will find it advantageous to consider a decomposition of the solution component $\left[\omega, \omega_{t}\right]$ into $\omega=\omega^{(1)}+\omega^{(2)}+\omega^{(3)}$ (again with the corresponding initial data $\left.\left[\omega_{0}, \omega_{1}\right] \in D\left(\mathcal{A}_{\gamma}^{2}\right)\right)$, where the $\omega^{(i)}$ solve, respectively,

$$
\begin{aligned}
& \left\{\begin{array}{l}
-\gamma \Delta \omega_{t t}^{(1)}+\Delta^{2} \omega^{(1)}=-\alpha \Delta \theta \text { on }(0, \infty) \times \Omega \\
\omega^{(1)}=\frac{\partial \omega^{(1)}}{\partial \nu}=0 \text { on }(0, \infty) \times \Gamma_{0} \\
\left\{\begin{array}{l}
\Delta \omega^{(1)}+(1-\mu) B_{1} \omega^{(1)}+\alpha \theta=0 \\
\frac{\partial \Delta \omega^{(1)}}{\partial \nu}+(1-\mu) \frac{\partial B_{2} \omega^{(1)}}{\partial \tau}-\gamma \frac{\partial \omega_{t t}^{(1)}}{\partial \nu}=0 \\
\omega^{(1)}(t=0)=\omega_{t}^{(1)}(t=0)=0
\end{array} \text { on }(0, \infty) \times \Gamma_{1}\right.
\end{array}\right. \\
& \left\{\begin{array}{l}
-\gamma \Delta \omega_{t t}^{(2)}+\Delta^{2} \omega^{(2)}=-\omega_{t t} \text { on }(0, \infty) \times \Omega ; \\
\omega^{(2)}=\frac{\partial \omega^{(2)}}{\partial \nu}=0 \text { on }(0, \infty) \times \Gamma_{0} \\
\left\{\begin{array}{l}
\Delta \omega^{(2)}+(1-\mu) B_{1} \omega^{(2)}=0 \\
\frac{\partial \Delta \omega^{(2)}}{\partial \nu}+(1-\mu) \frac{\partial B_{2} \omega^{(2)}}{\partial \tau}-\gamma \frac{\partial \omega_{t t}^{(2)}}{\partial \nu}+\alpha \frac{\partial \theta}{\partial \nu}=0
\end{array} \text { on }(0, \infty) \times \Gamma_{1} ;\right. \\
\omega^{(2)}(t=0)=\omega_{t}^{(2)}(t=0)=0 .
\end{array}\right. \\
& \left\{\begin{array}{l}
-\gamma \Delta \omega_{t t}^{(3)}+\Delta^{2} \omega^{(3)}=0 \text { on }(0, \infty) \times \Omega \\
\omega^{(3)}=\frac{\partial \omega^{(3)}}{\partial \nu}=0 \text { on }(0, \infty) \times \Gamma_{0} \\
\left\{\begin{array}{l}
\Delta \omega^{(3)}+(1-\mu) B_{1} \omega^{(3)}=0 \\
\frac{\partial \Delta \omega^{(3)}}{\partial \nu}+(1-\mu) \frac{\partial B_{2} \omega^{(3)}}{\partial \tau}-\gamma \frac{\partial \omega_{t t}^{(3)}}{\partial \nu}=0 \text { on }(0, \infty) \times \Gamma_{1} \\
\omega^{(3)}(0)=\omega^{0} ; \quad \omega_{t}^{(3)}(0)=\omega^{1}
\end{array}\right.
\end{array}\right.
\end{aligned}
$$

Through a semigroup formulation, the well posedness of (2.50) and (2.51) can be handled just as easily as the entire system (1.1); to wit, defining on the state space

\footnotetext{
${ }^{2}$ Notice that at this point, one might be tempted to straightaway majorize $\int_{0}^{T} a\left(D \gamma_{0} \omega, \omega\right) d t$ so as to obtain something like $\left|\int_{0}^{T} a\left(D \gamma_{0} \omega, \omega\right)\right| d t \leq C \int_{0}^{T}\left\|\AA^{1 / 2} \omega(t)\right\|_{L^{2}(\Omega)}^{2} d t$. However, this will not suffice as we do not have control over the constant $C(C$ may not be small $<<1)$. Therefore, we need a different, more complex argument which will culminate in the estimate (2.72) below; likewise for the term $\left|\int_{0}^{T}\left(\Delta \omega, \frac{\partial D \gamma_{0} \omega}{\partial \nu}\right)_{L^{2}\left(\Gamma_{0}\right)} d t\right|$.
} 
$D\left(\AA^{1 / 2}\right) \times H_{\Gamma_{0}, \gamma}^{1}(\Omega)$ the operator $\widetilde{\mathcal{A}}_{\gamma}$ as

$$
\widetilde{\mathcal{A}}_{\gamma} \equiv\left(\begin{array}{cc}
0 & \mathbf{I} \\
-\widetilde{P}_{\gamma}^{-1} \AA & 0
\end{array}\right)
$$

$$
\text { (where } \widetilde{P}_{\gamma} \equiv \gamma A_{N} \in \mathcal{L}\left(H_{\Gamma_{0}, \gamma}^{1}(\Omega), H_{\Gamma_{0}, \gamma}^{-1}(\Omega)\right) \text { ) }
$$$$
\text { with domain } D\left(\widetilde{\mathcal{A}}_{\gamma}\right)=\left\{\left[\omega_{1}, \omega_{2}\right] \in D\left(\AA^{\frac{3}{4}}\right) \times D\left(\AA^{\frac{1}{2}}\right)\right\} ;
$$

then with the same degree of effort as in the proof of Theorem 1.1, we can show that $\widetilde{\mathcal{A}}_{\gamma}$ generates a unitary $C_{0}$-group $\left\{e^{\widetilde{\mathcal{A}}_{\gamma} t}\right\}_{t \geq 0}$ on $D\left(\AA^{1 / 2}\right) \times H_{\Gamma_{0}, \gamma}^{1}(\Omega)$ (note we are using the knowledge that $\widetilde{P}_{\gamma}^{-1}$ exists, inasmuch as $A_{N}$ is elliptic on $H_{\Gamma_{0}, \gamma}^{1}(\Omega)$, and that $\widetilde{P}_{\gamma}=\gamma\left(\Delta+\AA G_{2} \gamma_{1}\right)$ from (1.29)). Consequently we have that $\omega^{(2)} \in$ $C\left([0, T] ; D\left(\AA^{1 / 2}\right) \times H_{\Gamma_{0}, \gamma}^{1}(\Omega)\right)$, with this unique solution of $(2.50)$ written explicitly as

$$
\left[\begin{array}{c}
\omega^{(2)}(t) \\
\omega_{t}^{(2)}(t)
\end{array}\right]=\int_{0}^{t} e^{\widetilde{\mathcal{A}}_{\gamma}(t-s)}\left[\begin{array}{c}
0 \\
\widetilde{P}_{\gamma}^{-1}\left(-\omega_{t t}(s)+\alpha \lambda \AA G_{2} \gamma_{0} \theta(s)\right)
\end{array}\right] d s
$$

where again $\omega_{t t}$ is the second time derivative of the solution component $\omega$. Recall that we are taking the initial data $\left[\omega^{0}, \omega^{1}, \theta^{0}\right]$ to be in $D\left(\mathcal{A}_{\gamma}^{2}\right)$, and so $\omega_{t t} \in$ $C\left([0, T] ; H_{\Gamma_{0}, \gamma}^{1}(\Omega)\right)$. Moreover, for arbitrary initial data, $\theta \in L^{2}\left(0, T ; H^{1}(\Omega) \cap\right.$ $\left.L_{\sigma+\lambda}^{2}(\Omega)\right)$, by Lemma 2.2 , and this regularity, coupled with the facts contained in (1.17), (1.19), and (1.4), provide that $\AA G_{2} \gamma_{0} \theta(t) \in L^{2}\left(0, T ; H_{\Gamma_{0}, \gamma}^{-1}(\Omega)\right)$. Hence the formula (2.55) is well defined. Likewise, $\omega^{(3)} \in C\left([0, T] ; D\left(\AA^{1 / 2}\right) \times H_{\Gamma_{0}, \gamma}^{1}(\Omega)\right)$ with

$$
\omega^{(3)}(t)=e^{\widetilde{\mathcal{A}}_{\gamma} t}\left[\begin{array}{c}
\omega^{0} \\
\omega^{1}
\end{array}\right] .
$$

Regarding the well posedness of the system (2.49), we have the following result from [14] and [13].

REGUlARITY TheOREM. For arbitrary initial data $\left[\omega_{0}, \omega_{1}\right] \in D\left(\AA^{1 / 2}\right) \times H_{\Gamma_{0}, \gamma}^{1}(\Omega)$, parameter $\xi \geq 0, f \in L^{2}\left(0, T ; H_{\Gamma_{0}, \gamma}^{-1}(\Omega)\right)$, and $g \in L^{2}\left(0, T ; H^{1 / 2}\left(\Gamma_{1}\right)\right)$, the following system is well posed:

$$
\left\{\begin{array}{l}
\xi \omega_{t t}-\gamma \Delta \omega_{t t}+\Delta^{2} \omega=f \text { on }(0, \infty) \times \Omega ; \\
\omega=\frac{\partial \omega}{\partial \nu}=0 \text { on }(0, \infty) \times \Gamma_{0} ; \\
\left\{\begin{array}{l}
\Delta \omega+(1-\mu) B_{1} \omega=g \\
\frac{\partial \Delta \omega}{\partial \nu}+(1-\mu) \frac{\partial B_{2} \omega}{\partial \tau}-\gamma \frac{\partial \omega_{t t}}{\partial \nu}=0
\end{array} \text { on }(0, \infty) \times \Gamma_{1} ;\right. \\
\omega(0)=\omega_{0}, \omega_{t}(0)=\omega_{1},
\end{array}\right.
$$

with the solution $\left[\omega, \omega_{t}\right] \in C\left([0, T] ; D\left(\AA^{1 / 2}\right) \times H_{\Gamma_{0}, \gamma}^{1}(\Omega)\right)$.

To make use of the above theorem for the resolution of (2.49) with arbitrary $\theta$ in $H^{1}(\Omega) \cap L_{\sigma+\lambda}^{2}(\Omega)$ subject to Robin boundary conditions, we note that $-\Delta=$ 
$A_{R}-\frac{\sigma}{\eta} \in \mathcal{L}\left(H^{1}(\Omega),\left[H^{1}(\Omega)\right]^{\prime}\right)$ and consequently $\Delta \theta \in L^{2}\left(0, T ; H_{\Gamma_{0}, \gamma}^{-1}(\Omega)\right)$; moreover, $\left.\theta\right|_{\Gamma} \in L^{2}\left(0, T ; H^{1 / 2}(\Gamma)\right)$ by the trace theorem, and so the regularity theorem will give us that

$$
\omega^{(1)} \in C\left([0, T] ; D\left(\AA^{\frac{1}{2}}\right) \times H_{\Gamma_{0}, \gamma}^{1}(\Omega)\right),
$$

with the pointwise estimate

$$
\begin{aligned}
\left\|\begin{array}{c}
\omega^{(1)}(t) \\
\omega_{t}^{(1)}(t)
\end{array}\right\|_{D\left(\AA^{\frac{1}{2}}\right) \times H_{\Gamma_{0}, \gamma}^{1}(\Omega)}^{2} & \leq C\left[\int_{0}^{T}\|\Delta \theta(t)\|_{H_{\Gamma_{0}, \gamma}^{-1}(\Omega)}^{2} d t+\alpha \int_{0}^{T}\|\theta(t)\|_{H^{\frac{1}{2}}\left(\Gamma_{1}\right)}^{2} d t\right] \\
& \leq C \int_{0}^{T}\|\theta(t)\|_{H^{1}(\Omega) \cap L_{\sigma+\lambda}^{2}(\Omega)}^{2} d t .
\end{aligned}
$$

A simple uniqueness argument which makes use of the regularity theorem verifies that indeed the solution component $\omega \equiv \omega^{(1)}+\omega^{(2)}+\omega^{(3)}$. Moreover, concerning the explicit representation (2.55), an integration by parts has that

$$
\begin{aligned}
& \int_{0}^{t} e^{\widetilde{\mathcal{A}}_{\gamma}(t-s)}\left[\begin{array}{c}
0 \\
\widetilde{P}_{\gamma}^{-1} \omega_{t t}(s)
\end{array}\right] d s=\left.e^{\widetilde{\mathcal{A}}_{\gamma}(t-s)}\left[\begin{array}{c}
0 \\
\widetilde{P}_{\gamma}^{-1} \omega_{t}(s)
\end{array}\right]\right|_{0} ^{t} \\
& +\int_{0}^{t} e^{\widetilde{\mathcal{A}}_{\gamma}(t-s)} \widetilde{\mathcal{A}}_{\gamma}\left[\begin{array}{c}
0 \\
\widetilde{P}_{\gamma}^{-1} \omega_{t}(s)
\end{array}\right] d s \\
= & \left.e^{\widetilde{\mathcal{A}}_{\gamma}(t-s)}\left[\begin{array}{c}
0 \\
\widetilde{P}_{\gamma}^{-1} \omega_{t}(s)
\end{array}\right]\right|_{0} ^{t}-\int_{0}^{t} e^{\widetilde{\mathcal{A}}_{\gamma}(t-s)}\left[\begin{array}{c}
\widetilde{P}_{\gamma}^{-1} \omega_{t}(s) \\
0
\end{array}\right] d s,
\end{aligned}
$$

where the last equality above makes sense pointwise in $\left[D\left(\widetilde{\mathcal{A}}_{\gamma}^{*}\right)\right]^{\prime}=\left[D\left(\AA^{\frac{3}{4}}\right)\right]^{\prime} \times$ $\left[D\left(\AA^{1 / 2}\right)\right]^{\prime}$; hence upon majorizing (2.55) with the expression (2.60) in mind (and using the contraction of the semigroup $\left.\left\{e^{\widetilde{\mathcal{A}}_{\gamma}(t)}\right\}_{t \geq 0}\right)$, we have

$$
\left\|\begin{array}{c}
\omega^{(2)}(t) \\
\omega_{t}^{(2)}(t)
\end{array}\right\|_{D\left(\AA^{\frac{1}{2}}\right) \times H_{\Gamma_{0}, \gamma}^{1}(\Omega)}^{2} \leq C_{T}\left[\|\theta\|_{L^{2}\left(0, T ; H^{1}(\Omega) \cap L_{\sigma+\lambda}^{2}(\Omega)\right)}^{2}+\left\|\omega_{t}\right\|_{C\left([0, T] ; L^{2}(\Omega)\right)}^{2}\right] .
$$

Thus, using (2.59), (2.61), and the explicit representation (2.56), we have

$$
\begin{aligned}
& \left\|\left[\begin{array}{c}
\omega^{(1)}(t)+\omega^{(2)}(t) \\
\omega^{(1)}(t)+\omega_{t}^{(2)}(t)
\end{array}\right]\right\|_{D\left(\AA^{\frac{1}{2}}\right) \times H_{\Gamma_{0}, \gamma}^{1}(\Omega)}^{2} \\
& \leq C_{T}\left[\|\theta\|_{L^{2}\left(0, T ; H^{1}(\Omega) \cap L_{\sigma+\lambda}^{2}(\Omega)\right)}^{2}+\left\|\omega_{t}\right\|_{C\left([0, T] ; L^{2}(\Omega)\right)}^{2}\right] ; \\
& \left\|\left[\begin{array}{c}
\omega^{(3)}(t) \\
\omega_{t}^{(3)}(t)
\end{array}\right]\right\|_{D\left(\AA^{\frac{1}{2}}\right) \times H_{\Gamma_{0}, \gamma}^{1}(\Omega)}^{2} \leq E_{\gamma}(0) .
\end{aligned}
$$

Further analyzing $\omega^{(3)}$, if we make the substitution $z \equiv \Delta \omega^{(3)}$, we then note that $z$ solves the wave equation

$$
\gamma z_{t t}=\Delta z
$$


with $\left[z, z_{t}\right] \in C\left([0, T] ; L^{2}(\Omega) \times H^{-1}(\Omega)\right)$. Consequently, the recent regularity result of [26] (specifically, apply Theorem 3 therein together with Remark 2.3 and the remark after Theorem 9 in [26]) reveals that $z$ has a "trace" on $\Gamma$ with a positive constant $C(T, \gamma)$ and a $\rho>0$ such that the following estimate holds: ${ }^{3}$

$$
\left\|\left.z\right|_{\Gamma}\right\|_{L^{2}\left(0, T ; H^{-\frac{1}{2}+\rho}(\Gamma)\right)} \leq C(T, \gamma)\left\|\left[z, z_{t}\right]\right\|_{C\left([0, T] ; L^{2}(\Omega) \times H^{-1}(\Omega)\right)}
$$

and as pointwise we have

$$
\|z(t)\|_{L^{2}(\Omega)}^{2}+\left\|z_{t}(t)\right\|_{H^{-1}(\Omega)}^{2} \leq C E_{\gamma}(0)
$$

(from the estimate (2.63)), we end up with

$$
\left\|\left.\Delta \omega^{(3)}\right|_{\Gamma}\right\|_{L^{2}\left(0, T ; H^{-\frac{1}{2}+\rho}(\Gamma)\right)}^{2} \leq C(T, \gamma) E_{\gamma}(0) .
$$

Recall that $\omega^{(3)}$, as the solution of (2.51), satisfies

$$
\Delta \omega^{(3)}-(1-\mu) \frac{\partial^{2} \omega^{(3)}}{\partial \tau^{2}}=(1-\mu) \kappa \frac{\partial \omega^{(3)}}{\partial \nu} \text { on }(0, T) \times \Gamma_{1},
$$

where $\kappa$ denotes the curvature, and so (2.68), coupled with the estimates (2.67) and

$$
\left\|\frac{\partial \omega^{(3)}}{\partial \nu}\right\|_{C\left([0, T] ; H^{\frac{1}{2}}\left(\Gamma_{1}\right)\right)} \leq C\left\|\omega^{(3)}\right\|_{C\left([0, T] ; H^{2}(\Omega)\right)} \leq C(T) E_{\gamma}(0),
$$

gives that $\frac{\partial^{2} \omega^{(3)}}{\partial \tau^{2}} \in L^{2}\left(0, T ; H^{-\frac{1}{2}+\rho}\left(\Gamma_{1}\right)\right)$ with

$$
\left\|\frac{\partial^{2} \omega^{(3)}}{\partial \tau^{2}}\right\|_{L^{2}\left(0, T ; H^{-\frac{1}{2}+\rho}\left(\Gamma_{1}\right)\right)} \leq C(T, \gamma) E_{\gamma}(0),
$$

and (2.69) is in turn equivalent to

$$
\left\|\gamma_{0} \omega^{(3)}\right\|_{L^{2}\left(0, T ; H^{\frac{3}{2}+\rho}\left(\Gamma_{1}\right)\right)} \leq C(T, \gamma) E_{\gamma}(0) .
$$

REMARK 6. The estimate in (2.70) can also be derived independently of Tataru's result in [26] by decomposing problem (2.51) microlocally into respective elliptic and hyperbolic parts. In the elliptic sector, we can use standard elliptic regularity and the boundary conditions on $\Gamma_{1}$ to deduce the regularity of the trace $\gamma_{0} \omega^{(3)}$ in $H^{2}\left(0, T \times \Gamma_{1}\right)$. In the hyperbolic sector, we apply the transformation $z \equiv \Delta \omega^{(3)}$, and we are subsequently led to the study of the wave equation with its forcing term in $L^{2}\left(0, T ; H^{-1}(\Omega)\right)$ (due to microlocalization). The arguments presented in [16] and (see also [17]) apply to the hyperbolic sector specifically and provide the estimate (2.70) valid in that sector. Combining elliptic and hyperbolic estimates yields (2.70) with the value of $\rho$ being at least $\frac{1}{10}$. Instead, the estimate obtained by using Tataru's result [26] leads to the optimal value of $\rho=\frac{1}{6}$.

\footnotetext{
${ }^{3}$ We note that the value of $\rho$ depends on the geometry; however, we always have $\rho>0$.
} 
Given this extra regularity for the trace of $\left.\omega^{(3)}\right|_{\Gamma_{1}}$, we can hence invoke a classical PDE interpolation inequality to finally obtain

$$
\begin{aligned}
& \left\|\gamma_{0} \omega^{(3)}\right\|_{L^{2}\left(0, T ; H^{\frac{3}{2}}\left(\Gamma_{1}\right)\right)}^{2} \leq C(T, \gamma)^{-1}\left\|\gamma_{0} \omega^{(3)}\right\|_{L^{2}\left(0, T ; H^{\frac{3}{2}+\rho}\left(\Gamma_{1}\right)\right)}^{2} \\
& \quad+C_{T, \gamma}\left\|\gamma_{0} \omega^{(3)}\right\|_{L^{2}\left(0, T ; H^{\frac{1}{2}}\left(\Gamma_{1}\right)\right)}^{2}
\end{aligned}
$$

(where $C(T, \gamma)$ is as in (2.70), and $C_{T, \gamma}$ denotes another positive constant depending on $T$ and $\gamma$ )

$\leq E_{\gamma}(0)+C_{T, \gamma}\left\|\omega^{(3)}\right\|_{L^{2}\left(0, T ; H^{1}(\Omega)\right)}^{2}$

(after using the estimate (2.70) and trace theory)

$$
\leq E_{\gamma}(0)+C_{T, \gamma}\|\omega\|_{L^{2}\left(0, T ; H^{1}(\Omega)\right)}^{2}+C_{T, \gamma}\left\|\omega^{(1)}+\omega^{(2)}\right\|_{L^{2}\left(0, T ; H^{1}(\Omega)\right)}^{2}
$$

(after using the decomposition $\omega=\omega^{(1)}+\omega^{(2)}+\omega^{(3)}$ )

$$
\leq E_{\gamma}(0)+C_{T, \gamma}\left[\|\theta\|_{L^{2}\left(0, T ; H^{1}(\Omega) \cap L_{\sigma+\lambda}^{2}(\Omega)\right)}^{2}+\|\omega\|_{L^{2}\left(0, T ; H^{1}(\Omega)\right)}^{2}+\left\|\omega_{t}\right\|_{C\left([0, T] ; L^{2}(\Omega)\right)}^{2}\right],
$$

after using the inequality (2.62).

With the decomposition of $\omega$ in hand, along with its accompanying norm estimates, particularly that of the trace $\gamma_{0} \omega^{(3)}$ in $(2.71)$, we can now deal with the recalcitrant terms $\left|\int_{0}^{T} a\left(D \gamma_{0} \omega, \omega\right) d t\right|$ and $\left|\int_{0}^{T}\left(\Delta \omega, \frac{\partial D \gamma_{0} \omega}{\partial \nu}\right)_{L^{2}\left(\Gamma_{0}\right)} d t\right|$ :

(A5.i) Dealing with $\left|\int_{0}^{T} a\left(D \gamma_{0} \omega, \omega\right) d t\right|$ :

$$
\begin{aligned}
& \left|\int_{0}^{T} a\left(D \gamma_{0} \omega, \omega\right) d t\right| \\
= & \left|\int_{0}^{T} a\left(D \gamma_{0}\left(\omega^{(1)}+\omega^{(2)}+\omega^{(3)}\right), \omega\right) d t\right| \\
\leq & \int_{0}^{T} C\left\|D \gamma_{0}\left(\omega^{(1)}+\omega^{(2)}+\omega^{(3)}\right)\right\|_{H^{2}(\Omega)}\left\|\AA^{\frac{1}{2}} \omega\right\|_{L^{2}(\Omega)} d t
\end{aligned}
$$

(after using the fact that $D \gamma_{0} \in \mathcal{L}\left(H^{2}(\Omega)\right)$ )

$$
\begin{aligned}
\leq & \epsilon \int_{0}^{T}\|\AA \omega\|_{L^{2}(\Omega)}^{2} d t+C_{T, \gamma}\left[\int_{0}^{T}\|\theta\|_{H^{1}(\Omega) \cap L^{2}(\Omega)}^{2} d t+\|\omega\|_{L^{2}\left(0, T ; H^{1}(\Omega)\right)}^{2}\right. \\
& \left.+\left\|\omega_{t}\right\|_{C\left([0, T] ; L^{2}(\Omega)\right)}^{2}\right]
\end{aligned}
$$

$$
+C\left[E_{\gamma}(T)+E_{\gamma}(0)\right],
$$

after using the boundedness of the Dirichlet map $D$ followed by the standard mean inequality as well as the crucial estimates (2.71) and (2.62) (here we have not noted the noncrucial dependence of $\epsilon$ in the constant $\left.C_{T, \gamma}\right)$.

(A.5ii) Dealing with $\left|\int_{0}^{T}\left(\Delta \omega, \frac{\partial D \gamma_{0} \omega}{\partial \nu}\right)_{L^{2}\left(\Gamma_{0}\right)} d t\right|$. By Lemma $2,\left.\Delta \omega\right|_{\Gamma_{0}} \in L^{2}\left(0, T ; L^{2}\left(\Gamma_{0}\right)\right)$, and so with this bit of information we have

$$
\begin{aligned}
& \left|\int_{0}^{T}\left(\Delta \omega, \frac{\partial D \gamma_{0} \omega}{\partial \nu}\right)_{L^{2}\left(\Gamma_{0}\right)} d t\right| \\
\leq & C \int_{0}^{T}\|\Delta \omega\|_{L^{2}\left(\Gamma_{0}\right)}\left\|D \gamma_{0} \omega\right\|_{H^{2}(\Omega)} d t
\end{aligned}
$$

(by the trace theorem) 


$$
\begin{aligned}
= & C \int_{0}^{T}\|\Delta \omega\|_{L^{2}\left(\Gamma_{0}\right)}\left\|D \gamma_{0}\left(\omega^{(1)}+\omega^{(2)}+\omega^{(3)}\right)\right\|_{H^{2}(\Omega)} d t \\
\leq & \frac{\epsilon}{C} \int_{0}^{T}\|\Delta \omega\|_{L^{2}\left(\Gamma_{0}\right)}^{2} d t+C_{T, \gamma}\left[\int_{0}^{T}\|\theta\|_{H^{1}(\Omega) \cap L^{2}(\Omega)}^{2} d t+\|\omega\|_{L^{2}\left(0, T ; H^{1}(\Omega)\right)}^{2}\right. \\
& \left.+\left\|\omega_{t}\right\|_{C\left([0, T] ; L^{2}(\Omega)\right)}^{2}\right] \\
& +C\left[E_{\gamma}(T)+E_{\gamma}(0)\right]
\end{aligned}
$$

(again using the mean inequality followed by (2.71) and (2.62),

and where the inverted positive constant $C$ is that in (2.24))

$$
\leq \epsilon \int_{0}^{T}\left[\left\|\AA^{\frac{1}{2}} \omega\right\|_{L^{2}(\Omega)}^{2}+\int_{0}^{T}\left\|P_{\gamma}^{\frac{1}{2}} \omega\right\|_{L^{2}(\Omega)}^{2}\right] d t+C_{T, \gamma}\left[\int_{0}^{T}\|\theta\|_{H^{1}(\Omega) \cap L^{2}(\Omega)}^{2} d t\right.
$$

Combining (2.48), (2.72), and (2.73), we finally have

$$
\begin{aligned}
& \left(\frac{\alpha}{\beta}-3 \epsilon\right) \int_{0}^{T}\left[\left\|\omega_{t}\right\|_{L^{2}(\Omega)}^{2}+\gamma\left\|\nabla \omega_{t}\right\|_{L^{2}(\Omega)}^{2}\right] d t \\
\leq & 3 \epsilon \int_{0}^{T}\left\|\AA^{\frac{1}{2}} \omega\right\|_{L^{2}(\Omega)}^{2} d t+C_{T, \gamma}\left[\int_{0}^{T}\|\theta\|_{H^{1}(\Omega) \cap L_{\bar{\sigma}+\lambda}^{2}(\Omega)}^{2} d t\right. \\
& \left.+\|\omega\|_{L^{2}\left(0, T ; H^{1}(\Omega)\right)}^{2}+\left\|\omega_{t}\right\|_{C\left([0, T] ; L^{2}(\Omega)\right)}^{2}\right] \\
& +C\left[E_{\gamma}(0)+E_{\gamma}(T)\right] .
\end{aligned}
$$

(B) Conclusion of the Proof of Theorem 3. To majorize the norm of the component $\omega$, we multiply (1.35) by $\omega$, integrate from 0 to $T$ and employ Green's theorem to obtain (after accounting for the boundary conditions and using (1.20))

$$
\begin{gathered}
\left.\left(P_{\gamma}^{\frac{1}{2}} \omega_{t}, P_{\gamma}^{\frac{1}{2}} \omega\right)_{L^{2}(\Omega)}\right|_{0} ^{T}-\int_{0}^{T}\left\|P_{\gamma}^{\frac{1}{2}} \omega_{t}\right\|_{L^{2}(\Omega)}^{2} d t \\
=-\int_{0}^{T}\left\|\AA^{\frac{1}{2}} \omega\right\|_{L^{2}(\Omega)}^{2} d t-\alpha \int_{0}^{T}\left(\theta, \frac{\partial \omega}{\partial \nu}\right)_{L^{2}\left(\Gamma_{1}\right)} d t \\
+\alpha \int_{0}^{T}(\nabla \theta, \nabla \omega)_{L^{2}(\Omega)} d t ;
\end{gathered}
$$

since by the trace theorem we have pointwise

$$
\begin{aligned}
& \left|\left(\theta, \frac{\partial \omega}{\partial \nu}\right)_{L^{2}\left(\Gamma_{1}\right)}\right|+\left|(\nabla \theta, \nabla \omega)_{L^{2}(\Omega)}\right| \\
\leq & C\left[\|\theta\|_{H^{\frac{1}{2}}(\Gamma)}\left\|\frac{\partial \omega}{\partial \nu}\right\|_{H^{\frac{1}{2}\left(\Gamma_{1}\right)}}+\|\theta\|_{H^{1}(\Omega)}\|\omega\|_{H^{1}(\Omega)}\right] \\
\leq & C\|\theta\|_{H^{1}(\Omega)}\|\omega\|_{H^{2}(\Omega)} \leq \epsilon\left\|\AA^{\frac{1}{2}} \omega\right\|_{L^{2}(\Omega)}^{2}+C_{\epsilon}\|\theta\|_{H^{1}(\Omega) \cap L_{\sigma+\lambda}^{2}(\Omega)}^{2},
\end{aligned}
$$

we thus arrive at the following. 
There exists a constant $C>0$ such that for $\epsilon>0$ small enough, the solution $\left[\omega, \omega_{t}, \theta\right]$ of $(1.1)$ satisfies

$$
\begin{aligned}
(1-\epsilon) \int_{0}^{T}\left\|\AA^{\frac{1}{2}} \omega\right\|_{L^{2}(\Omega)}^{2} d t \leq & C \int_{0}^{T}\left[\left\|\omega_{t}\right\|_{L^{2}(\Omega)}^{2}+\gamma\left\|\nabla \omega_{t}\right\|_{L^{2}(\Omega)}^{2}\right] d t \\
& +C\left(\int_{0}^{T}\|\theta\|_{H^{1}(\Omega) \cap L_{\sigma+\lambda}^{2}(\Omega)}^{2} d t+E_{\gamma}(T)+E_{\gamma}(0)\right),
\end{aligned}
$$

where the noncrucial dependence of $C$ upon $\epsilon$ has not been noted.

Thus, if $\epsilon$ is small enough, we then have, upon combining (2.74) and (2.77), the existence of constants $C$ and $C_{T, \gamma}$ such that

$$
\begin{aligned}
& \int_{0}^{T}\left[\left\|\AA^{\frac{1}{2}} \omega\right\|_{L^{2}(\Omega)}^{2}+\left\|\omega_{t}\right\|_{L^{2}(\Omega)}^{2}+\gamma\left\|\nabla \omega_{t}\right\|_{L^{2}(\Omega)}^{2}+\|\theta\|_{L^{2}(\Omega)}^{2}\right] d t \\
\leq & C_{T, \gamma} \int_{0}^{T}\|\theta\|_{H^{1}(\Omega) \cap L_{\sigma+\lambda}^{2}(\Omega)}^{2} d t+C\left[E_{\gamma}(T)+E_{\gamma}(0)\right] \\
& +C_{T, \gamma}\left[\|\omega\|_{L^{2}\left(0, T ; H^{1}(\Omega)\right)}^{2}+\left\|\omega_{t}\right\|_{C\left([0, T] ; L^{2}(\Omega)\right)}^{2}\right] .
\end{aligned}
$$

From here, we apply the relation (2.21) and its inherent dissipativity property (that is, $\left.E_{\gamma}(T) \leq E_{\gamma}(t) \forall 0 \leq t \leq T\right)$ to (2.78) to finally attain the preliminary inequality; namely, for $T>2 C$ (with $C$ as in (2.78) independent of $T$ ),

$$
\begin{aligned}
E_{\gamma}(T) \leq & \frac{C_{T, \gamma}+2 C \eta}{T-2 C} \int_{0}^{T}\|\theta\|_{H^{1}(\Omega) \cap L_{\sigma+\lambda}^{2}(\Omega)}^{2} d t \\
& +C_{T, \gamma}\left[\|\omega\|_{L^{2}\left(0, T ; H^{1}(\Omega)\right)}^{2}+\left\|\omega_{t}\right\|_{C\left([0, T] ; L^{2}(\Omega)\right)}^{2}\right] .
\end{aligned}
$$

A straightforward compactness-uniqueness argument similar to that employed in [15] and [1] will subsequently eliminate the lower order terms in (2.79), viz. we have the following proposition.

PROPOSITION 2.4. The presence of the inequality (2.79) implies that there exists a constant $C_{T}$ which satisfies

$$
\|\omega\|_{L^{2}\left(0, T ; H^{1}(\Omega)\right)}^{2}+\left\|\omega_{t}\right\|_{C\left([0, T] ; H^{2}(\Omega)\right)}^{2} d t \leq C_{T} \int_{0}^{T}\|\theta\|_{H^{1}(\Omega) \cap L_{\sigma+\lambda}^{2}(\Omega)}^{2} d t .
$$

Hence, the inequalities (2.79) and (2.80) give the desired estimate (2.36) (and consequently (2.35)), and so the proof of Theorem 1.3 is now complete.

Note added in proof. As one reads through the arguments in the present paper, he or she gathers the understanding that the key ingredient in our stability proof is the selection of the "right" multiplier $A_{D}^{-1} \theta$ (which is novel when compared to the standard differential multipliers used in plate theory). This multiplier was first devised in our paper [3] (which initially considered the easier case of the thermoelastic plate with lower order "clamped" or "hinged" boundary conditions), and we have since invoked it in later problems (see [4], [13], [6], [5]. In particular, [4] is a preliminary version 
of our present paper). In our present paper, it is this particular choice of multiplier which allows us to obtain sharp results on the uniform stabilization of thermoelastic plates with the higher order "free" boundary conditions in place, results which include the attainment of explicit decay rates.

Related work on this problem includes that of E. Bisognin, V. Bisognin, P. Menzala, and E. Zuazua in [7], who employed an alternative and indirect argument for the stabilization of the nonlinear thermoelastic plate in the case of clamped/hinged boundary conditions only. This method, even in the case of linear models, yielded weaker results than those posted in [2], [3]. (We assume that at the time of their work the four authors were unaware of [3].) Indeed, the indirect (proof by contradiction) method in [7] has the following shortcomings:

(i) The method requires two different treatments of the problem, corresponding to the respective cases $\gamma>0$ and $\gamma=0$. This dichotomy is necessitated by the fact that the accompanying decay rates they obtain blow up as $\gamma \downarrow 0$.

(ii) The decay rates they obtain are not explicit.

(iii) In the specific case $\gamma=0$, the analyticity of the underlying semigroup is used in an essential way, which precludes the possibility that their indirect method can be adjusted so as to give a unified treatment of the problem for all cases $\gamma \geq 0$ (recall that $\gamma>0$ corresponds to hyperbolic-like dynamics).

In contrast, the paper [3] (which is critical and constitutes a basis for the present paper) obtains decay estimates which are uniform in the parameter $\gamma \geq 0$, this being accomplished via the use of the multiplier $A_{D}^{-1} \theta$. As the authors of [7] were apparently informed much before the date of submission of [23] of this comparison between their work and that in [3] (this is a documented fact), one may then view as perplexing the subsequent appearance of the paper [23], which now claims for itself the right (and much improved with respect to [7]) result using the very same techniques and ideas as in [3] (which again are radically different from those in [7]). In particular, [23] uses the same multiplier and the same trace result, the latter being proclaimed therein as "hidden regularity." Perhaps adding to the perplexity is the fact that the two authors in [23], while freely addressing the aforementioned shortcomings of [7], make neither acknowledgment nor reference to [3]. Our main point here is to stress the fact that the critical multiplier and the resulting technique for proving uniform decay rates for thermoelastic plates takes its origin in [2], [3], and not in [23].

\section{REFERENCES}

[1] G. Avalos, The exponential stability of a coupled hyperbolic/parabolic system arising in structural acoustics, Abstract Appl. Anal., 1 (1996), pp. 203-219.

[2] G. Avalos And I. LASIECKA, Exponential stability of a thermoelastic system without mechanical dissipation, Rendiconti Di Instituto Di Matematica, an invited paper in a special issue honoring Pierre Grisvard; Rendiconti Di Instituto Di Matematica Dell'Universitá di Trieste, E. Mitidieri and Philippe Clement, eds., Suppl. Vol. XXVIII, 1997, p. 1-27.

[3] G. Avalos And I. Lasiecka, Exponential Stability of a Thermoelastic System without Mechanical Dissipation, IMA Preprint Series \#1357, November 1995.

[4] G. Avalos And I. LasieckA, Exponential Stability of a Simply Supported Thermoelastic System without Mechanical Dissipation II: The Case of Simply Supported Boundary Conditions, IMA Preprint \# 1397, March, 1996.

[5] G. Avalos And I. Lasiecka, Uniform decay rates for a nonlinear thermoelastic system, in Proc. European Control Conference, Brussels, Belgium, 1997.

[6] G. Avalos AND I. LAsiecKA, Uniform decay rates in nonlinear thermoelastic systems without mechanical dissipation, in Proc. IFIP WG 7.2 Conference on Optimal Control: Theory, Algorithms, and Applications, Gainesville, FL, Kluwer Academic Publishers, Norwell, MA, February 1997. 
[7] E. Bisognin, V. Bisognin, G. Perla Menzala, and E. Zuazua, On the Exponential Stability for von Karman Equations in the Presence of Thermal Effects, Preprint, 1996.

[8] P. Grisvard, Caracterization de quelques espaces d'interpolation, Arch. Rational Mech. Anal., 25 (1967), pp. 40-63.

[9] S. HANSEn, Boundary control of a one-dimensional linear thermoelastic rod, SIAM J. Control Optim., 32 (1994), pp. 1052-1074.

[10] J. U. KIM, On the energy decay of a linear thermoelastic bar and plate, SIAM J. Math. Anal., 23 (1992), pp. 889-899.

[11] V. Komornik, Exact controllability and stabilization: The multiplier method, Research in Applied Mathematics, John Wiley \& Sons, New York, 1994.

[12] J. Lagnese, Boundary Stabilization of Thin Plates, SIAM Stud. Appl. Math. 10, SIAM, Philadelphia, PA, 1989.

[13] I. LASIECKA, Control and stabilization of interactive structures, in Systems and Control in the Twenty-First Century, Birkhäuser Boston, Boston, MA, 1997, pp. 245-263.

[14] I. LASIECKA AND R. TRIGGiani, Exact controllability and uniform stabilization of Kirchoff plates with boundary control only on $\left.\Delta \omega\right|_{\Sigma}$ and homogeneous boundary displacement, J. Differential Equations, 88 (1991), pp. 62-101.

[15] I. LASIECKA AND R. TRIGGiani, Uniform stabilization of the wave equation with Dirichlet or Neumann feedback control without geometrical conditions, Appl. Math. Optim., 25 (1992), pp. 189-224.

[16] I. Lasiecka And R. TRIGgiani, Sharp regularity theory for second-order hyperbolic equations of Neumann type, Annali Mat. Pura \& Appl. IV, 207 (1990), pp. 285-367.

[17] I. LASIECKA AND R. TRIGGIANI, Recent advances in regularity of second-order hyperbolic mixed problems and applications, in Dynamics Reported, Expositions in Dynamical Systems, C. K. R. T. Jones, U. Kirchgraber, and H. O. Wather, eds., Springer-Verlag, New York, 1994, pp. 104-162.

[18] J.L. Lions, Contrôlabilité exacte, perturbations et stabilization de systèmes distribués, Vol. 1, Masson, Paris, 1989.

[19] J. L. Lions And E. Magenes, Non-Homogeneous Boundary Value Problems and Applications, Vol. 1, Springer-Verlag, New York, 1972.

[20] Z. Liu And M. RenARdy, A note on the equations of a thermoelastic plate, Appl. Math. Lett., 8 (1995), pp. 1-6.

[21] Z. Liu AND S. Zheng, Exponential stability of the Kirchoff plate with thermal or viscoelastic damping, Quarterly Appl. Math., 55 (1997), pp. 551-564.

[22] Z. LiU AND S. Zheng, Exponential stability of semigroup associated with a thermal elastic system, Quarterly Appl. Math., 52 (1993), pp. 535-545.

[23] G. Perla Menzala and E. Zuazua, Explicit exponential decay rates for solutions of von Karman's system of thermoelastic plates, C.R. Acad. Sci. Paris, 324 (1997), pp. 49-54.

[24] K. NARUKAwA, Boundary value control of thermoelastic systems, Hiroshima Math. J., 13 (1983), pp. 227-272.

[25] J. E. M. RiverA, Energy decay rate in linear thermoelasticity, Funkcial. Ekvac., 35 (1992), pp. 19-30.

[26] D. TAtaru, On the Regularity of Boundary Traces for the Wave Equation, Ann. Scuóla Normale, to appear.

[27] I. LAsieckA and R. Triggiani, Analyticity and lack thereof, of thermo-elastic semigroups, in Proc. Conference on Control of PDEs, CIRM, Luminy, France, June 1997. 